\title{
BLOWN-UP INTERSECTION COCHAINS AND DELIGNE'S SHEAVES
}

\author{
DAVID CHATAUR, MARTINTXO SARALEGI-ARANGUREN, AND DANIEL TANRÉ
}

\begin{abstract}
In a series of papers the authors introduced the so-called blown-up intersection cochains. These cochains are suitable to study products and cohomology operations of intersection cohomology of stratified spaces. The aim of this paper is to prove that the sheaf versions of the functors of blown-up intersection cochains are realizations of Deligne's sheaves. This proves that Deligne's sheaves can be incarnated at the level of complexes of sheaves by soft sheaves of perverse differential graded algebras. We also study Poincaré and Verdier dualities of blown-up intersections sheaves with the use of Borel-Moore chains of intersection.
\end{abstract}

\section{CONTENTS}

1. Pseudomanifolds and perversities

2. Blown-up intersection cohomology

3. Intersection chains and cochains 11

4. Poincaré and Verdier dualities 19

References

Let $R$ be a commutative unitary ring and let $M$ be an oriented topological manifold of dimension $n$, without boundary, but not necessarily compact. Poincaré duality is satisfied as an isomorphism

$$
H^{*}(M ; R) \cong H_{n-*}^{\mathrm{BM}}(M ; R)
$$

between singular cohomology and Borel-Moore homology (see [2]). There is also a compact supports version which is an isomorphism

$$
H_{c}^{*}(M ; R) \cong H_{n-*}(M ; R)
$$

between singular cohomology with compact supports and singular homology. These two isomorphisms come from the cap product with a fundamental class $[M] \in H_{n}^{\mathrm{BM}}(M ; R)$.

Date: May 8, 2019.

2010 Mathematics Subject Classification. 55N33, 14F05, 14F43, 55M05, 57P10, 55U30.

Key words and phrases. Intersection homology; Deligne sheaf; Verdier duality; Poincaré duality; Blown-up cohomology.

This research was supported through the program "Research in Pairs" at the Mathematisches Forschunginstitut Oberwolfach in 2016. The authors thank the MFO for its generosity and hospitality. The third author was also supported by the MINECO and FEDER research project MTM2016-78647-P. 
Poincaré duality and sheaf theory. Let us recall how to interpret these isomorphisms in terms of complexes of sheaves of $R$-modules, where $R$ is a principal ideal domain. We consider two sheaves, $\mathbf{N}^{*}$ and $\mathbf{C}_{\mathrm{BM}}^{*}$. The sheaf of singular cochains $\mathbf{N}^{*}$ is the sheafification of the presheaf of singular cochains

$$
U \mapsto N^{*}(U ; R) .
$$

The sheaf of Borel-Moore chains $\mathbf{C}_{\mathrm{BM}}^{*}$ is the sheafification of the presheaf of relative locally finite singular chains ([13, section 3.1])

$$
U \mapsto C_{n-*}^{\mathrm{BM}}(M, M \backslash \bar{U} ; R) .
$$

These two sheaves are both acyclic resolutions of the constant sheaf $\underline{\mathbf{R}}$. In the derived category $\mathcal{D}(M)$ of complexes of sheaves of $R$-modules we have the isomorphisms,

$$
\underline{\mathbf{R}} \cong \mathbf{N}^{*} \cong \mathbf{C}_{\mathrm{BM}}^{*} .
$$

Then taking hypercohomology we have the following isomorphisms,

$$
H^{*}(M ; R) \cong \mathbb{H}^{*}(M ; \underline{\mathbf{R}}) \cong \mathbb{H}^{*}\left(M ; \mathbf{N}^{*}\right) \cong \mathbb{H}^{*}\left(M ; \mathbf{C}_{\mathrm{BM}}^{*}\right) \cong H_{n-*}^{\mathrm{BM}}(M ; R),
$$

and the corresponding compact supports version,

$$
H_{c}^{*}(M ; R) \cong \mathbb{H}_{c}^{*}(M ; \underline{\mathbf{R}}) \cong \mathbb{H}_{c}^{*}\left(M ; \mathbf{N}^{*}\right) \cong \mathbb{H}_{c}^{*}\left(M ; \mathbf{C}_{\mathrm{BM}}^{*}\right) \cong H_{n-*}(M ; R) .
$$

Let $\mathbf{F}^{*}$ be a complex of sheaves on $M$. Borel, Moore and Verdier introduced a dual sheaf $\mathbb{D}\left(\mathbf{F}^{*}\right)$ (see $[1, \mathrm{~V} .7]$ ) which gives an isomorphism

$$
\mathbb{D}\left(\mathbf{C}_{\mathrm{BM}}^{*}[n]\right) \cong \mathbf{N}^{*}
$$

in the derived category $\mathcal{D}(M)$. This proves that the constant sheaf $\underline{\mathbf{R}}$ is self-dual for Verdier duality:

$$
\mathbb{D}(\underline{\mathbf{R}}[n]) \cong \underline{\mathbf{R}} .
$$

As a consequence, we get a bilinear pairing

$$
H^{i}(M ; R) \otimes H_{c}^{n-i}(M ; R) \rightarrow R
$$

between singular cohomology and singular cohomology with compact supports that is nonsingular on the torsion free parts of $H^{i}(M ; R)$ and $H_{c}^{n-i}(M ; R)$.

Poincaré duality and singular spaces. For singular spaces, Poincaré duality is not satisfied in general. However when the space is stratified, Verdier duality still makes sense and we can ask for the existence of self-dual sheaves.

In the case of an oriented pseudomanifold $X$ of dimension $n$, Goresky and MacPherson introduced complexes of sheaves called intersection complexes $\mathbf{I C}_{\bar{p}}^{*}$ parametrized by sequences of integers called perversities $\bar{p}([17,18])$. For a fixed perversity $\bar{p}$ they give a list of axioms, the Deligne's axioms, that characterize $\mathbf{I C}_{\bar{p}}^{*}$ up to isomorphism in the derived category $\mathcal{D}(X)$. Working over a field $\mathbb{F}$, they proved the following duality result

$$
\mathbb{D}\left(\mathbf{I C}_{\bar{p}}^{*}[n]\right) \cong \mathbf{I} \mathbf{C}_{D \bar{p}}^{*}
$$

where $D \bar{p}$ is the complementary perversity of $\bar{p}$. As a consequence, in cohomology, we get a nonsingular bilinear pairing

$$
\mathbb{H}^{i}\left(X ; \mathbf{I C}_{\bar{p}}^{*}\right) \otimes \mathbb{H}_{c}^{n-i}\left(X ; \mathbf{I C}_{D \bar{p}}^{*}\right) \rightarrow \mathbb{F} .
$$


This pairing comes from an intersection type product

$$
\mathbf{I C}_{\bar{p}}^{i} \otimes \mathbf{I C}_{\bar{q}}^{j} \rightarrow \mathbf{I C}_{\bar{p}+\bar{q}}^{i+j}
$$

defined in the derived category $\mathcal{D}(X)$.

Blown-up cochains. In $[9,6,8]$ we introduced a functor of singular cochains $\widetilde{N}_{\bar{p}}^{*}$, the blown-up cochains (also called Thom-Whitney cochains in the first works). In fact, we have a family of cochain complexes $\left\{\widetilde{N}_{\bar{p}}^{*}(X ; R)\right\}_{\bar{p} \in \mathcal{P}}$ compatible with the poset of perversities $\mathcal{P}$ that comes equipped with a cup product,

$$
\widetilde{N}_{\bar{p}}^{i}(X ; R) \otimes \widetilde{N}_{\bar{q}}^{j}(X ; R) \stackrel{-\cup-}{\longrightarrow} \widetilde{N}_{\bar{p}+\bar{q}}^{i+j}(X ; R),
$$

whose construction is related to the classical cup product on the singular cochain complex $N^{*}(X ; R)$.

There is also the singular chain complex $C_{*}^{\bar{p}}(X ; R)$ introduced by King in [21] whose sheafification is the intersection sheaf of Goresky and MacPherson. The homology of $C_{*}^{\bar{p}}(X ; R)$ is called intersection homology. Let us notice that to intersect chains in $C_{*}^{\bar{p}}(X ; R)$ one generally needs to deform them, the cup product defined on $\widetilde{N}_{\bar{p}}^{*}(X ; R)$ obviates this requirement. Moreover, in [8] we introduced a cap product between $\widetilde{N}_{\bar{p}}^{*}(X ; R)$ and a generalization $\mathfrak{C}_{*}^{\bar{p}}(X ; R)$ of $C_{*}^{\bar{p}}(X ; R)$, better adapted to large perversities, see Definition 3.3. When $X$ is compact and oriented, we proved that the cap product with a cycle $\gamma_{X} \in \mathfrak{C}_{n}^{\overline{0}}(X ; R)$ representing the fundamental class $\Gamma_{X} \in \mathfrak{H}_{n}^{\overline{0}}(X ; R)$,

$$
\widetilde{N}_{\bar{p}}^{i}(X ; R) \stackrel{-\cap \gamma_{X}}{\longrightarrow} \mathfrak{C}_{n-i}^{\bar{p}}(X ; R),
$$

is a quasi-isomorphism for any ring $R$ ([10, Theorem $\mathrm{B}])$. This extends the Poincaré duality theorem of Friedman and McClure [16] established for field coefficients.

Outline. In this paper we study the sheafification $\widetilde{\mathbf{N}}_{\bar{p}}^{*}$ (see Definition 2.5) of the blownup cochains $\widetilde{N}_{\bar{p}}^{*}$. After a brief recall in Section 1 of the basic definitions, Theorem A of Section 2 is our first result: the sheaf $\widetilde{\mathbf{N}}_{\bar{p}}^{*}$ satisfies Deligne's axioms. As a consequence, we get that $\widetilde{\mathbf{N}}_{\bar{p}}^{*}$ offers a way to promote the multiplicative structure of Deligne's sheaves directly on the complex of sections of $\widetilde{\mathbf{N}}_{\bar{p}}^{*}$. This multiplicative enrichment at the level of sections has some nice consequences for the study of intersection cohomology: it makes the development of a rational homotopy theory possible in this context ([9]) and brings a better paradigm for the study of Steenrod squares in intersection cohomology ([6]).

Let $R$ be a principal ideal domain and $I_{R}^{*}$ an injective resolution of $R$. In Section 3, we introduce a complex of Borel-Moore chains of intersection, denoted by $\mathfrak{C}_{*}^{\mathrm{BM}, \bar{p}}(X ; R)$. In the case of a PL-pseudomanifold, we prove (see Proposition 3.11):

Proposition A. Let $X$ be a paracompact PL-pseudomanifold with a perversity $\bar{p}$. Then, there exists a quasi-isomorphism, linear for the structure of perverse $\widetilde{N}_{\bar{q}}^{*}(X ; R)$-modules,

$$
\Phi_{X}: \mathfrak{C}_{k}^{\mathrm{BM}, \bar{p}}(X ; R) \rightarrow \operatorname{Hom}_{k}\left(\mathfrak{C}_{\bar{p}, c}^{*}(X ; R), I_{R}^{*}\right) .
$$

Thus $\operatorname{Hom}_{k}\left(\mathfrak{C}_{\bar{p}, c}^{*}(X ; R), I_{R}^{*}\right)$ appears as the bidual of the complex of intersection chains. 
In Section 4, we build a diagram of cochain complexes,

$$
\widetilde{N}_{\bar{p}}^{k}(X ; R) \stackrel{\mathcal{D}_{X}}{\longrightarrow} \operatorname{Hom}_{n-k}\left(\mathfrak{C}_{\bar{p}, c}^{*}(X ; R), I_{R}^{*}\right)
$$

where

- $D P_{X}$ is the cap product with a fundamental cycle $\gamma_{X} \in \mathfrak{C}_{n}^{\mathrm{BM}, \overline{0}}(X ; R)$,

- $\Phi_{X}$ is the morphism of Proposition A,

- $\mathcal{D}_{X}$ is a product with the cocycle $\Phi_{X}\left(\gamma_{X}\right)$.

This diagram relates directly at the chain level Poincaré and Verdier dualities and we prove (see Proposition 4.4 and Corollary 4.5):

Proposition B. The previous diagram is commutative and all maps are linear quasiisomorphisms for the structure of perverse $\widetilde{N}_{\bar{q}}^{*}(X ; R)$-modules.

We fix for the sequel a principal ideal domain $R$. For a topological space $X$, we denote by $\stackrel{\circ}{\mathrm{C}}=X \times[0,1[/ X \times\{0\}$ the open cone on $X$. The apex of the cone is $\mathrm{v}=[-, 0]$.

\section{Pseudomanifolds And Perversities}

This section is a recall of definitions of basic objects: pseudomanifolds and perversities. We consider perversities defined on each stratum which cover the original case of Goresky and MacPherson.

\subsection{Topological background.}

Definition 1.1. A filtered space $\left(X,\left(X_{i}\right)_{0 \leq i \leq n}\right)$, is a Haussdorff topological space $X$ equipped with a filtration by closed subspaces,

$$
X_{0} \subseteq X_{1} \subseteq \cdots \subseteq X_{n}=X
$$

such that the complement, $X_{n} \backslash X_{n-1}$, is non-empty. The integer $d(X)=n$ is the formal dimension of $X$. The connected components, $S$, of $X_{i} \backslash X_{i-1}$ are the strata of $X$ and we set $d(S)=i$. The strata of $X_{n} \backslash X_{n-1}$ are called regular, the other being called singular. We denote by $\mathcal{S}_{X}$ the set of non-empty strata of $X$. The sub-space $X_{n-1}$ is the singular set also denoted by $\Sigma_{X}$.

Definition 1.2. A stratified topological pseudomanifold or pseudomanifold for short, of dimension $n$ is a filtered topological space $\left(X,\left(X_{i}\right)_{0 \leq i \leq n}\right)$ such that, for any $i$, $X_{i} \backslash X_{i-1}$ is a topological manifold of dimension $i$ or the empty set. Moreover, for any point $x \in X_{i} \backslash X_{i-1}, i \neq n$, we have:

(1) an open neighbourhood, $V$, of $x \in X$, together with the induced filtration $V_{i}=V \cap X_{i}$

(2) an open neighbourhood, $U$, of $x \in X_{i} \backslash X_{i-1}$,

(3) a compact pseudomanifold $L$ of dimension $n-i-1$, called the link of $x$, the cone ${ }_{c}^{\circ} L$ being endowed with the conical filtration $(\stackrel{\circ}{c} L)_{i}=\stackrel{\circ}{c}\left(L_{i-1}\right)$, 
(4) a homeomorphism $\varphi: U \times \stackrel{\circ}{c} L \rightarrow V$ such that:

- $\varphi(u, \mathrm{v})=u$, for all $u \in U$,

- $\varphi\left(U \times \stackrel{\circ}{c} L_{j}\right)=V \cap X_{i+j+1}$, for all $j \in\{0, \ldots, n-i-1\}$.

The pair $(\varphi, V)$ is called a local chart. A pseudomanifold $X$ is $R$-oriented if its regular part $X \backslash \Sigma$ is $R$-oriented.

Definition 1.3. Let $\left(X,\left(X_{i}\right)_{0 \leq i \leq n}\right)$ and $\left(Y,\left(Y_{i}\right)_{0 \leq i \leq n}\right)$ be pseudomanifolds. A stratum preserving map is a continuous map $f: X \rightarrow Y$ such that for any stratum $S \in \mathcal{S}_{X}$ there exists a stratum $S^{f} \in \mathcal{S}_{Y}$ such that $f(S) \subset S^{f}$ and $\operatorname{codim}\left(S^{f}\right)=\operatorname{codim}(S)$.

\subsection{Perversities and perverse spaces.}

Definition 1.4. A perversity on a filtered topological space $\left(X,\left(X_{i}\right)_{0 \leq i \leq n}\right)$, is a function, $\bar{p}: \mathcal{S}_{X} \rightarrow \mathbb{Z}$, defined on the set of strata of $X$ and such that $\bar{p}(S)=0$ on any regular stratum $S$. The couple $(X, \bar{p})$ is called a perverse space. If $X$ is a pseudomanifold, we say a perverse pseudomanifold. We denote by $\mathcal{P}$ the set of perversities on $X$.

Let us review some important perversities.

(1) A GM perversity (for Goresky and Mac-Pherson, see [17]) is a sequence of integers $\bar{p}:\{0, \ldots, n\} \rightarrow \mathbb{N}$ verifying $\bar{p}(0)=\bar{p}(1)=\bar{p}(2)=0$ and $\bar{p}(i) \leq \bar{p}(i+1) \leq$ $\bar{p}(i)+1$. It induces a perversity as above, defined as $\bar{p}(S)=\bar{p}(\operatorname{codim}(S))$.

(2) The zero perversity $\overline{0}$ is the GM perversity defined as $\overline{0}(i)=0$ for any $i \in$ $\{0, \ldots, n\}$.

(3) The top perversity $\bar{t}$ is the GM perversity given by $\bar{t}(i)=i-2$ for $i \geq 2$. We say that two perversities $\bar{p}$ and $\bar{q}$ are complementary if for any singular stratum $S$ we have:

$$
\bar{p}(S)+\bar{q}(S)=\bar{t}(S)=n-\operatorname{dim}(S)-2 .
$$

Each perversity $\bar{p}$ has a complementary perversity $D \bar{p}$ defined by $D \bar{p}=\bar{t}-\bar{p}$. When $\bar{p}$ is a GM perversity $D \bar{p}$ is also GM.

Definition 1.5. Let $f: X \rightarrow Y$ be a stratum preserving map and $\bar{q}$ a perversity on $Y$. We define the pull-back perversity $f^{*} \bar{q}$ thanks to the formula:

$$
f^{*} \bar{q}(S)=\bar{q}\left(S^{f}\right)
$$

for any $S \in S_{X}$.

Example 1.6. Let us review the three fundamental examples that we encounter in this paper.

(1) Let $U \subset X$ be an open set of a pseudomanifold, endowed with the induced filtration. The inclusion $i: U \hookrightarrow X$ is a stratum preserving map. By abuse of notation, the pull-back perversity $i^{*} \bar{p}$ is also denoted by $\bar{p}$.

(2) Let $M$ be an $m$-dimensional manifold and $(X, \bar{p})$ be a pseudomanifold. The product filtration on $X \times M$ is defined by $(X \times M)_{i}=X_{i-m} \times M$. The projection map $\pi: X \times M \rightarrow X$ is a stratum preserving morphism. By abuse of notation, the pull-back perversity $\pi^{*} \bar{p}$ will also be denoted by $\bar{p}$.

(3) Let $L$ be a compact filtered space. A perversity $\bar{p}$ on the cone ${ }^{\circ} L$ is determinate by the number $\bar{p}(\mathrm{v})$ and a perversity on $L$, still denoted by $\bar{p}$. They are related by the formula $\bar{p}(S)=\bar{p}(S \times] 0,1[)$, for each $S \in \mathcal{S}_{L}$. 
1.3. Perverse structures. Unlike singular cohomology that comes equipped with a cup product, intersection cohomology at a fixed perversity is not an algebra. Rather it is parametrized by a family of perversities, and it has a product that is compatible with perversities. This has been formalized by M. Hovey who introduced perverse chain complexes, perverse differential graded algebras in [19]. Let us review this formalism. It relies on the fact that the set of perversities has two remarkable features.

(1) The set $\mathcal{P}$ of perversities on a filtered space $X$ is a poset: we say that $\bar{p} \geq \bar{q}$ if for any stratum $S$ we have $\bar{p}(S) \geq \bar{q}(S)$.

(2) We can add perversities: $(\bar{p}+\bar{q})(S)=\bar{p}(S)+\bar{q}(S)$. And the zero perversity $\overline{0}$ is a unit for this addition.

Thanks to these two properties we define perverse chain complexes, perverse algebras.

Definition 1.7. Let $(\mathcal{M}, \square, \mathbb{I})$ be a symmetric monoidal category.

(1) A perverse object in $\mathcal{M}$ is a functor $M_{\bullet}: \mathcal{P} \rightarrow \mathcal{M}$. That is, we have for each perversity $\bar{p}$ an object $M_{\bar{p}}$ of $\mathcal{M}$ and a collection of morphisms of $\mathcal{M}$,

$$
\gamma_{\bar{p} \leq \bar{q}}: M_{\bar{p}} \rightarrow M_{\bar{q}},
$$

whenever $\bar{p} \leq \bar{q}$ such that $\gamma_{\bar{p} \leq \bar{p}}=\mathrm{id}$ and $\gamma_{\bar{q} \leq \bar{r}} \circ \gamma_{\bar{p} \leq \bar{q}}=\gamma_{\bar{p} \leq \bar{r}}$.

(2) A perverse monoid in $\mathcal{M}$ is a perverse object $M_{\bullet}$ together with a unit $\eta: \mathbb{I} \rightarrow M_{\overline{0}}$ and associative products,

$$
\mu: M_{\bar{p}} \square M_{\bar{q}} \rightarrow M_{\bar{p}+\bar{q}},
$$

compatible with the poset structure. A perverse monoid in the category of complexes is called a perverse differential graded algebra.

(3) A (left) perverse module over a perverse monoid $A_{\bullet}$ is a perverse object $M_{\bullet}$ together with products

$$
\perp: A_{\bar{p}} \square M_{\bar{q}} \rightarrow M_{\bar{p}+\bar{q}}
$$

compatible with the poset structure and satisfying the classical properties of modules.

Example 1.8. We will consider the next examples of categories $\mathcal{M}$.

- The category of graded $R$-modules: intersection homology and cohomogy provide examples of perverse objects in this category.

- The category of complexes of $R$-modules: the blown-up cochains introduced in the next section, equipped with its cup product is a perverse monoid.

- The category of complexes of sheaves of $R$-modules on $X$.

\section{BLOWN-UP INTERSECTION COHOMOLOGY}

In this section we introduce the cochain complex of blown-up intersection cochains $\widetilde{N}_{\bar{p}}^{*}$ and prove, in Theorem A, that its sheafification $\widetilde{\mathbf{N}}_{\mathbf{p}}^{*}$ gives a nice realization of the Deligne sheaf.

2.1. Blown-up intersection cochains. We start with the standard $k$-simplex viewed as an ordered simplicial complex $\Delta$. We associate to this $k$-simplex the simplicial chain complex $N_{*}(\Delta)$, whose component of $l$-chains is the $R$-module generated by the $l$-faces of $\Delta$, together with the usual differential given by the face operators. We define $N^{*}(\Delta)$ as the $R$-linear dual of $N_{*}(\Delta)$. This simplicial complex has a geometric realization which 
is the standard $k$-simplex embedded in $\mathbb{R}^{k+1}$, by abuse of notations we also denote this geometric realization as $\Delta$.

Our constructions use simplices in a "good" position with respect to the filtration of $X$, let us give a precise definition.

Definition 2.1. Let $(X, \bar{p})$ be a perverse space.

(i) A filtered simplex $\sigma: \Delta \rightarrow X$ is a singular simplex such that each $\sigma^{-1}\left(X_{i}\right)$ is a face of $\Delta$. A filtered simplex admits a join decomposition:

$$
\Delta=\Delta_{0} * \cdots * \Delta_{n} .
$$

A filtered simplex is said to be regular if $\Delta_{n} \neq \emptyset$.

(ii) Let $F \operatorname{Simp}(X)$ be the category whose objects are the regular simplices of $X$ and whose morphisms are induced by the face operators of the simplices.

If $\sigma: \Delta \rightarrow X$ is a regular simplex and $F$ a regular face of $\Delta$, we notice that the canonical map $i: F \rightarrow \Delta$ induces a morphism in $F \operatorname{Simp}(X)$ between $\sigma \circ i$ and $\sigma$.

Having defined filtered simplices we can introduce the functor of blown-up cochains as a system of coefficients on the category $F \operatorname{Simp}(X)$.

Definition 2.2. Let $(X, \bar{p})$ be a perverse space and $\sigma: \Delta_{0} * \cdots * \Delta_{n} \rightarrow X$ a fixed regular simplex.

(i) To $\sigma$ we associate the cochain complex:

$$
\widetilde{N}_{\sigma}^{*}=N^{*}\left(\mathrm{c} \Delta_{0}\right) \otimes N^{*}\left(\mathrm{c} \Delta_{1}\right) \otimes \cdots \otimes N^{*}\left(\mathrm{c} \Delta_{n-1}\right) \otimes N^{*}\left(\Delta_{n}\right) .
$$

(ii) For any regular face $F$ of $\sigma$ we have the restriction morphism:

$$
i^{*}: \widetilde{N}_{\sigma}^{*} \rightarrow \widetilde{N}_{\sigma \circ i}^{*}
$$

We define the blown-up cochains as the inverse limit

$$
\widetilde{N}^{*}(X)=\lim _{\sigma \in F \operatorname{Simp}(X)} \widetilde{N}_{\sigma}^{*} .
$$

Said differently a blown-up cochain is a collection $\left\{\omega_{\sigma}\right\}_{\sigma: \Delta \rightarrow X}$ of tensor products of cochains parametrized by regular simplices, satisfying gluing conditions with respect to restrictions to the regular faces. A blown-up cochain owns a perverse degree relatively to any stratum of $X$. Let us see that.

Definition 2.3. Let $\Delta=\Delta_{0} * \cdots * \Delta_{n}$ be a regular simplex. For any $1 \leq k \leq n$ we consider the restriction map,

$\rho_{k}: N^{*}\left(\mathrm{c} \Delta_{0}\right) \otimes \cdots \otimes N^{*}\left(\mathrm{c} \Delta_{n-1}\right) \otimes N^{*}\left(\Delta_{n}\right) \rightarrow N^{*}\left(\mathrm{c} \Delta_{0}\right) \otimes \cdots \otimes N^{*}\left(\mathrm{c} \Delta_{n-k-1}\right) \otimes N^{*}\left(\Delta_{n-k}\right) \otimes$ $N^{*}\left(\mathrm{c} \Delta_{n-k+1}\right) \otimes \cdots \otimes N^{*}\left(\mathrm{c} \Delta_{n-1}\right) \otimes N^{*}\left(\Delta_{n}\right)$.

For each $\eta \in N^{*}\left(c \Delta_{0}\right) \otimes \cdots \otimes N^{*}\left(c \Delta_{n-1}\right) \otimes N^{*}\left(\Delta_{n}\right)$, the cochain $\rho_{k}(\eta)$ can be written as a finite $\operatorname{sum} \sum_{i \in I} a_{i} \otimes b_{i}$, where $\left\{a_{i}\right\}_{i \in I}$ is an $R$-basis of the free module $N^{*}\left(\mathrm{c} \Delta_{0}\right) \otimes$ $\cdots \otimes N^{*}\left(\Delta_{n-k}\right)$ and $b_{i} \in N^{*}\left(\mathrm{c} \Delta_{n-k+1}\right) \otimes \cdots \otimes N^{*}\left(\Delta_{n}\right)$. We set

$$
\|\eta\|_{k}= \begin{cases}-\infty & \text { if } \rho_{k}(\eta)=0 \\ \max \left\{\operatorname{deg}\left(b_{i}\right) \mid b_{i} \neq 0\right\} & \text { if } \rho_{k}(\eta) \neq 0\end{cases}
$$


Definition 2.4. Let $\omega$ be a cochain of $\widetilde{N}^{*}(X ; R)$. The perverse degree of $\omega$ along a singular stratum, $S \in \mathcal{S}_{X}$, is equal to

$$
\|\omega\|_{S}=\sup \left\{\left\|\omega_{\sigma}\right\|_{\operatorname{codim} S} \mid \sigma: \Delta \rightarrow X \text { regular with } \sigma(\Delta) \cap S \neq \emptyset\right\} .
$$

where $\sup \emptyset=-\infty$.

Let us fix a perversity $\bar{p}$ on $X$. We say that $\omega \in \widetilde{N}^{*}(X ; R)$ is $\bar{p}$-allowable if for any singular stratum $S$ we have $\|\omega\|_{S} \leq \bar{p}(S)$. The cochain $\omega$ is a $\bar{p}$-intersection cochain if $\omega$ and its coboundary, $\delta \omega$, are $\bar{p}$-allowable. We denote by $\widetilde{N}_{\bar{p}}^{*}(X ; R)$ the complex of $\bar{p}$ intersection cochains and by $\mathscr{H}_{\bar{p}}^{*}(X ; R)$ its homology, called blown-up $\bar{p}$-intersection cohomology of $X$ with coefficients in $R$, for the perversity $\bar{p}$.

2.2. Properties of blown-up cochains. Let us recall the main properties of blown-up cochains (see [8], [10] or [9], [6], [7] where they are called TW-cochains). They satisfy the Mayer-Vietoris property [8, Theorem $\mathrm{C}]$ and are natural with respect to stratum preserving morphisms (for a careful study of this naturality [8, Theorem A]).

We also have a stratified homotopy invariance $[8$, Theorem D]. If $(X, \bar{p})$ is a perverse space then the canonical projection $\mathrm{pr}: X \times \mathbb{R} \rightarrow X$, which is a stratum preserving map, induces a quasi-isomorphism

$$
\operatorname{pr}^{*}: \widetilde{N}_{\bar{p}}^{*}(X ; R) \rightarrow \widetilde{N}_{\bar{p}}^{*}(X \times \mathbb{R} ; R) .
$$

A crucial property is the computation of the blown-up intersection cohomology of an open cone. Let $L$ be a compact filtered topological space and $\bar{p}$ a perversity on the cone $\stackrel{\circ}{L}$, of apex v. We have [8, Theorem E]:

$$
\mathscr{H}_{\bar{p}}^{k}(\stackrel{\circ}{c} L ; R)= \begin{cases}\mathscr{H}_{\bar{p}}^{k}(L ; R), & \text { if } k \leq \bar{p}(\mathrm{v}), \\ 0 & \text { if } k>\bar{p}(\mathrm{v}) .\end{cases}
$$

If $k \leq \bar{p}(\mathrm{v})$, the previous isomorphism is given by the inclusion $L \times] 0,1[=\stackrel{\circ}{\mathrm{c}} L-\{\mathrm{v}\} \hookrightarrow \stackrel{\circ}{\mathrm{c}} L$.

Moreover, the collection $\left\{\widetilde{N}_{\bar{p}}^{*}(X ; R)\right\}_{\bar{p} \in \mathcal{P}}$ is a perverse differential graded algebra whose products,

$$
-\cup-: \widetilde{N}_{\bar{p}}^{*}(X ; R) \otimes \widetilde{N}_{\bar{q}}^{*}(X ; R) \longrightarrow \widetilde{N}_{\bar{p}+\bar{q}}^{*}(X ; R),
$$

are induced by the classical cup product on the tensor product $N^{*}\left(\mathrm{c} \Delta_{0}\right) \otimes \cdots \otimes N^{*}\left(\mathrm{c} \Delta_{n-1}\right) \otimes$ $N^{*}\left(\Delta_{n}\right)$ (see [8, Section 4]).

\subsection{The sheaf of blown-up intersection cochains.}

Definition 2.5. Let us consider an open covering $\mathcal{U}$ of $X$ the complex of small $\mathcal{U}$ cochains denoted by $\widetilde{N}_{\bar{p}}^{*}, \mathcal{U}(X ; R)$ is the chain complex of blown-up cochains defined on filtered simplices included in an open set of the covering $\mathcal{U}$, see [8, Definition 9.6]. If $\mathcal{U}^{\prime}$ is a covering that refines $\mathcal{U}$, we have canonical restriction maps

$$
\widetilde{N}_{\bar{p}}^{*}(X ; R) \rightarrow \widetilde{N}_{\bar{p}}^{*, \mathcal{U}}(X ; R) \rightarrow \widetilde{N}_{\bar{p}}^{*}, \mathcal{U}^{\prime}(X . R) .
$$

Let $V$ be an open subset of $X$. We define the category $\operatorname{Cov}(V)$ of open coverings of $V$ as the category associated to the poset of open coverings. The sheaf of blown-up 
intersection cochains $\widetilde{\mathbf{N}}_{\bar{p}}^{*}$ is defined by its sections as the direct limit

$$
\Gamma\left(V ; \widetilde{\mathbf{N}}_{\bar{p}}^{*}\right)=\lim _{\mathcal{U} \in \operatorname{Cov}(V)} \widetilde{N}_{\bar{p}}^{*, \mathcal{U}}(V ; R) .
$$

As the cup product is natural with restriction maps, the family $\left\{\widetilde{\mathbf{N}}_{\bar{p}}^{*}\right\}_{\bar{p} \in \mathcal{P}}$ is a perverse differential graded algebra.

Proposition 2.6. Let $(X, \bar{p})$ be a perverse space. The complex of sheaves $\widetilde{\mathbf{N}}_{\bar{p}}^{*}$ over $X$ is a complex of flat and soft sheaves.

Proof. (Flatness) Let us prove that for any $x \in X$ the stalk $\left(\widetilde{\mathbf{N}}_{\bar{p}}^{*}\right)_{x}$ is flat. A direct limit of flat modules being also flat, it suffices to prove that the module of sections $\Gamma\left(V ; \widetilde{\mathbf{N}}_{\bar{p}}^{*}\right)$ is a flat module for any open subset $V \subset X$. This module of sections is also the direct limit of small $\mathcal{U}$-cochains $\widetilde{N}_{\bar{p}}^{*}, \mathcal{U}(V ; R)$. We are therefore reduced to prove that these small cochains are flat. As we work over a principal ideal domain, a torsion free module is flat and a sub-module of a torsion free module is also torsion free. Thus let us use the fact that $\widetilde{N}_{\bar{p}}^{*}(V ; R) \subset \widetilde{N}^{*}(V ; R)$ and that $\widetilde{N}^{*}(V ; R)$ is $R$-torsion free.

(Softness) For any perversity $\bar{p}$ the complex of sheaves $\widetilde{\mathbf{N}}_{\bar{p}}^{*}$ is a complex of $\widetilde{\mathbf{N}}_{\overline{0}}^{*}$-modules, this structure being induced by the cup product $\widetilde{\mathbf{N}}_{0}^{*} \otimes \widetilde{\mathbf{N}}_{\bar{p}}^{*} \stackrel{-\cup-}{\longrightarrow} \widetilde{\mathbf{N}}_{\bar{p}}^{*}$. In particular they are $\tilde{\mathbf{N}}_{\overline{0}}^{0}$-modules.

We have constructed in [8, Lemma 10.2] an $R$-linear operator $N^{*}(X ; R) \rightarrow \widetilde{N}_{\overline{0}}^{*}(X ; R)$. As this operator preserves the product, we have a morphism of sheaves of rings $\mathbf{N}^{0} \rightarrow \widetilde{\mathbf{N}}_{0}^{0}$ between the singular zero cochains and the blown-up zero cochains in perversity $\overline{0}$. We recall that a complex of sheaves of modules over a soft sheaf of unital rings is soft. We conclude by using the fact that the sheaf $\mathbf{N}^{0}$ is soft.

Proposition 2.7. Let $(X, \bar{p})$ be a perverse space. We have the following isomorphisms

$$
\mathscr{H}_{\bar{p}}^{*}(X ; R) \cong H^{*}\left(\Gamma\left(X ; \widetilde{\mathbf{N}}_{\bar{p}}^{*}\right)\right) \cong \mathbb{H}^{*}\left(X ; \widetilde{\mathbf{N}}_{\bar{p}}^{*}\right) .
$$

Proof. The theorem of small $\mathcal{U}$-cochains (see [8, Corollary 9.8]) implies that for any open covering of $X$ the restriction map $\widetilde{N}_{\bar{p}}^{*}(X ; R) \rightarrow \widetilde{N}_{\bar{p}}^{*}, \mathcal{U}(X ; R)$ is a quasi-isomorphism. This gives us the first isomorphism, the second being a direct consequence of the softness of the sheaf $\widetilde{\mathbf{N}}_{\bar{p}}^{*}$.

\subsection{Blown-up intersection cochains with compact supports.}

Definition 2.8. Let $(X, \bar{p})$ be a perverse space. The blown-up complex of cochains with compact supports is the direct limit,

$$
\widetilde{N}_{\bar{p}, c}^{*}(X)=\lim _{K} \widetilde{N}_{\bar{p}}^{*}(X, X \backslash K ; R),
$$

where the limit is taken over all compact subsets $K \subset X$. Its cohomology is denoted by $\mathscr{H}_{\bar{p}, c}^{*}(X ; R)$.

Proposition 2.9. Let $(X, \bar{p})$ be a perverse space. We have the following isomorphisms

$$
\mathscr{H}_{\bar{p}, c}^{*}(X ; R) \cong H^{*}\left(\Gamma_{c}\left(X ; \widetilde{\mathbf{N}}_{\bar{p}}^{*}\right)\right) \cong \mathbb{H}_{c}^{*}\left(X ; \widetilde{\mathbf{N}}_{\bar{p}}^{*}\right) .
$$


Proof. The second isomorphism follows from the softness of the sheaf $\widetilde{\mathbf{N}}_{\bar{p}}^{*}$. In order to get the first one we use the fact that

$$
\Gamma_{c}\left(X ; \widetilde{\mathbf{N}}_{\bar{p}}^{*}\right)=\lim _{\mathcal{U} \in \operatorname{Cov}(X)} \widetilde{N}_{\bar{p}, c}^{*, \mathcal{U}}(X ; R)
$$

where $\widetilde{N}_{\bar{p}, c}^{*, \mathcal{U}}(X ; R)$ is the cochain complex of $\mathcal{U}$-small blown-up cochains with compact supports. And to conclude we use the theorem of small $\mathcal{U}$-cochains with compact supports proved in [10, Proposition 2.5].

2.5. Deligne's sheaves and blown-up cochains. Intersection homology was first introduced in [17] for PL-pseudomanifolds. In [18], Goresky and MacPherson replace the simplicial point of view of [17] by a sheaf construction due to Deligne [11]. Topological pseudomanifolds and GM perversities are the paradigm of [18], the main tool being an axiomatic characterization of the Deligne sheaf in the derived category $\mathcal{D}(X)$ of complexes of sheaves on $X$. If one replaces GM perversities by the perversities of Definition 1.4, an adaptation $\mathbf{Q}_{\bar{p}}^{*}$ of the Deligne's sheaf is done by G. Friedman in [15], thanks to a generalized truncation functor for sheaves.

Let $(X, \bar{p})$ be a perverse pseudomanifold. We show that the sheaf $\widetilde{\mathbf{N}}_{\bar{p}}^{*}$ is isomorphic to $\mathbf{Q}_{\bar{p}}^{*}$ in $\mathcal{D}(X)$. Let us first recall the Friedman version of the Deligne's axioms for perverse spaces (see [15, Section 3]).

Definition 2.10. Let $\mathbf{F}^{*}$ be a sheaf complex on $X$. We denote by $\mathbf{F}_{k}^{*}$ the restriction of $\mathbf{F}^{*}$ to $X \backslash X_{n-k}$. We say that $\mathbf{F}^{*}$ satisfies the axioms $(A X)_{\bar{p}}$ if:

(1) $\mathbf{F}^{*}$ is bounded, $\mathbf{F}^{i}=0$ for $i<0$ and the restriction $\mathbf{F}_{1}^{*}$ to the regular strata is quasi-isomorphic to the ordinary singular cochain complex.

(2) For any stratum $S$ and any $x \in S$, the cohomology sheaf $\mathbf{H}^{i}\left(\mathbf{F}^{*}\right)_{x}$ vanishes if $i>\bar{p}(S)$.

(3) For any stratum $S \subset X_{n-k}$ and any $x \in S$, the attachment map, $\alpha_{k}: \mathbf{F}_{k+1}^{*} \rightarrow$ $R i_{k}^{*} \mathbf{F}_{k}^{*}$, induced by the canonical inclusion $X \backslash X_{n-k} \hookrightarrow X \backslash X_{n-k-1}$ is a quasiisomorphism at $x$ up to $\bar{p}(S)$.

If $\mathbf{F}^{*}$ is soft, from [23, Remark 2.3], we may replace the axiom (3) by the following one.

(3 bis) For any $k \in\{2, \ldots, n\}$, stratum $S \subset X_{n-k} \backslash X_{n-k-1}, x \in S$ and $j \leq \bar{p}(S)$, the restriction map induces an isomorphism,

$$
\lim _{V_{x}} H^{j}\left(\Gamma\left(V_{x} ; \mathbf{F}^{*}\right)\right) \rightarrow \lim _{V_{x}} H^{j}\left(\Gamma\left(V_{x} \backslash X_{n-k} ; \mathbf{F}^{*}\right)\right),
$$

where $V_{x}$ varies into a cofinal family of neighborhoods of $x$ in $X \backslash X_{n-k-1}$.

Proposition 2.11. [15, Proposition 3.8] Let $(X, \bar{p})$ be a perverse pseudomanifold. Then, any sheaf complex satisfying the axioms $(A X)_{\bar{p}}$ is isomorphic to $\mathbf{Q}_{\bar{p}}^{*}$ in $\mathcal{D}(X)$.

In [14], G. Friedman extends such unicity theorem to the setting of the homotopically stratified spaces of F. Quinn, [24]. We do not consider these spaces in this work.

Our first result relates Deligne's sheaf to the blown-up intersection cohomology. 
Theorem A. Let $(X, \bar{p})$ be a perverse pseudomanifold. Then the sheaf complex $\widetilde{\mathbf{N}}_{\bar{p}}^{*}$ is isomorphic to $\mathbf{Q}_{\bar{p}}^{*}$ in the derived category $\mathcal{D}(X)$ of sheaves on $X$. Hence we have the following isomorphism:

$$
\mathscr{H}_{\bar{p}}^{*}(X ; R) \cong \mathbb{H}^{*}\left(X ; \mathbf{Q}_{\bar{p}}\right) .
$$

Moreover, this isomorphism is compatible with the products.

Proof. With Proposition 2.11, it is sufficient to prove that the sheaf $\widetilde{\mathbf{N}}_{\bar{p}}^{*}$ satisfies the axioms $(A X)_{\bar{p}}$.

Property (1) follows from the fact that the restriction of the sheaf $\widetilde{\mathbf{N}}_{\bar{p}}^{*}$ to the regular strata is the sheafification of the singular cochains.

The fact that $\widetilde{\mathbf{N}}_{\bar{p}}^{*}$ satisfies (2) and (3 bis) follows from the softness of this sheaf and the properties of blown-up intersection cohomology recalled in (2.2). Let us begin with axiom (2). Let $S$ be a stratum of $X_{n-k}$ and $x \in S$. We choose a cofinal family of local charts such that $\varphi: V_{x} \cong \mathbb{R}^{n-k} \times \stackrel{\circ}{\rightarrow} L$ where $L$ is the link of $x$. By softness of $\widetilde{\mathbf{N}}_{\bar{p}}^{*}$ we have the following isomorphisms:

$$
\mathbf{H}^{i}\left(\widetilde{\mathbf{N}}_{\bar{p}}^{*}\right)_{x} \cong \lim _{V_{x}} H^{i}\left(\Gamma\left(V_{x}, \widetilde{\mathbf{N}}_{\bar{p}}^{*}\right)\right) \cong \lim _{V_{x}} \mathscr{H}_{\bar{p}}^{i}\left(V_{x}, R\right) .
$$

Following (2.1) we get that

$$
\mathbf{H}^{i}\left(\widetilde{\mathbf{N}}_{\bar{p}}^{*}\right)_{x} \cong \mathscr{H}_{\bar{p}}^{i}\left(\mathbb{R}^{n-k} \times \stackrel{\circ}{\mathrm{c}} L ; R\right) \cong \mathscr{H}_{\bar{p}}^{i}(\stackrel{\circ}{\mathrm{c}} L ; R) .
$$

Then (2) follows from the fact that $\mathscr{H}_{\bar{p}}^{i}(\stackrel{\circ}{\mathrm{c}} L ; R)=0$ whenever $i>\bar{p}(S)$, cf. (2.2). In order to prove that $\widetilde{\mathbf{N}}_{\bar{p}}^{*}$ satisfies (3 bis), we use the same cofinal family of local charts. We have to analyze the attaching map $\mathscr{H}_{\bar{p}}^{i}\left(V_{x} ; R\right) \rightarrow \mathscr{H}_{\bar{p}}^{i}\left(V_{x} \backslash X_{n-k} ; R\right)$. Using $\varphi$, the attaching map becomes $\mathscr{H}_{\bar{p}}^{i}\left(\mathbb{R}^{n-k} \times \stackrel{\circ}{\mathrm{c}} L ; R\right) \rightarrow \mathscr{H}_{\bar{p}}^{i}\left(\mathbb{R}^{n-k} \times(\stackrel{\circ}{\mathrm{c}} L \backslash\{x\}) ; R\right)$. Now, the result is a consequence of (2.1) and (2.2).

The restriction of the product of blown-up cochains (see [8, Proposition 4.2]) on the regular part is the classical cup product, which is a resolution of the canonical product

$$
\underline{\mathbf{R}} \otimes \underline{\mathbf{R}} \rightarrow \underline{\mathbf{R}} .
$$

The result follows from a direct application of [15, Theorem 1.4], which asserts that this canonical product defined on the regular part of $X$ can be extended uniquely to the Deligne's sheaves.

Let us focus on the fact that the product on Deligne sheaves is only defined at the level of the derived category $\mathcal{D}(X)$, whereas the blown-up cochains give a sheaf of perverse algebras. This is the starting point of a rational homotopy theoretic treatment of intersection cohomology as done in [9].

\section{IntERSECTION CHAins AND COCHAins}

In this section we introduce the intersection chain complex and its dual, the intersection cochain complex, together with a Borel-Moore intersection chain complex. In Proposition 3.11, we extend to pseudomanifolds a theorem of Spanier ([26]) originally stated for singular chains and cochains on manifolds. 
3.1. Borel-Moore intersection chains. We denote by $C_{*}^{\mathrm{BM}}(X ; R)$ the complex of formal sums $\xi=\sum_{j} \lambda_{j} \sigma_{j}$, with $\sigma_{j}$ filtered, $\lambda_{j} \in R$ and such that any $x \in X$ possesses a neighboorhood for which the restriction of $\xi$ is finite. We say that $\xi$ is locally finite or a Borel-Moore chain. If we restrict to finite linear combinations, we recover the filtered chains and denote by $C_{*}(X ; R)$ the associated complex.

Definition 3.1. Consider a perverse space $(X, \bar{p})$ and a filtered simplex $\sigma: \Delta=\Delta_{0} *$ $\cdots * \Delta_{n} \rightarrow X$.

(i) The perverse degree of $\sigma$ is the $(n+1)$-tuple, $\|\sigma\|=\left(\|\sigma\|_{0}, \ldots,\|\sigma\|_{n}\right)$, where $\|\sigma\|_{i}=\operatorname{dim}\left(\Delta_{0} * \cdots * \Delta_{n-i}\right)$, with the convention $\operatorname{dim} \emptyset=-\infty$.

(ii) Given a stratum $S$ of $X$, the perverse degree of $\sigma$ along $S$ is defined by

$$
\|\sigma\|_{S}=\left\{\begin{array}{cc}
-\infty, & \text { if } S \cap \operatorname{Im} \sigma=\emptyset, \\
\|\sigma\|_{\text {codim } S} & \text { if } S \cap \operatorname{Im} \sigma \neq \emptyset .
\end{array}\right.
$$

(iii) The filtered singular simplex $\sigma$ is $\bar{p}$-allowable if $\|\sigma\|_{S} \leq \operatorname{dim} \Delta-\operatorname{codim} S+\bar{p}(S)$, for any stratum $S$.

(iv) A (finite or locally finite) chain $c$ is $\bar{p}$-allowable if it is a linear combination of $\bar{p}$-allowable simplices. The chain $c$ is a $\bar{p}$-intersection chain if $c$ and its boundary $\partial c$ are $\bar{p}$-allowable chains. The complexes of $\bar{p}$-intersection chains of $X$ with the differential $\partial$ are denoted by $C_{*}^{\bar{p}}(X ; R)$ or $C_{*}^{\mathrm{BM}, \bar{p}}(X ; R)$. Their respective homology $H_{*}^{\bar{p}}(X ; R)$ and $H_{*}^{\mathrm{BM}, \bar{p}}(X ; R)$ are respectively the $\bar{p}$-intersection homology and the Borel-Moore $\bar{p}$-intersection homology of $X$.

For a perversity $\bar{p}$ such that $\bar{p} \not \leq \bar{t}$, we may have $\bar{p}$-allowable simplices that are not regular in the sense of Definition 2.1. This failure has bad consequences on Poincaré duality (see [10]). To overcome this point, the tame intersection homology and cohomology has been introduced in [25] and [8]. Let us remind it.

Definition 3.2. Given a regular simplex $\Delta=\Delta_{0} * \cdots * \Delta_{n}$ we denote by $\mathfrak{d} \Delta$ the regular part of the chain $\partial \Delta$. That is $\mathfrak{d} \Delta=\partial\left(\Delta_{0} * \cdots * \Delta_{n-1}\right) * \Delta_{n}$, if $\left|\Delta_{n}\right|=0$, or $\mathfrak{d} \Delta=\partial \Delta$, if $\left|\Delta_{n}\right| \geq 1$.

Definition 3.3. Let $(X, \bar{p})$ be a perverse space. Given a regular simplex $\sigma: \Delta \rightarrow X$, we define the chain $\mathfrak{d} \sigma$ by $\sigma \circ \mathfrak{d}$. Notice that $\mathfrak{d}^{2}=0$. We denote by $\mathfrak{C}_{*}(X ; R)$ (resp. $\left.\mathfrak{C}_{*}^{\mathrm{BM}}(X ; R)\right)$ the complex of finite chains (resp. locally finite chains) generated by the regular simplices, endowed with the differential $\mathfrak{d}$.

A $\bar{p}$-allowable filtered simplex $\sigma: \Delta \rightarrow X$ is $\bar{p}$-tame if $\sigma$ is also a regular simplex. In the two cases, finite or locally finite, a chain $\xi$ is $\bar{p}$-tame if it is a linear combination of $\bar{p}$-tame simplices. A chain $\xi$ is a tame $\bar{p}$-intersection chain if $\xi$ and $\mathfrak{d} \xi$ are $\bar{p}$-tame chains.

We write $\mathfrak{C}_{*}^{\bar{p}}(X ; R) \subset \mathfrak{C}_{*}(X ; R)$ the complex of tame $\bar{p}$-intersection finite chains endowed with the differential $\mathfrak{d}$. Its homology $\mathfrak{H}_{*}^{\bar{p}}(X ; R)$ is the tame $\bar{p}$-intersection homology.

In the same manner, we write $\mathfrak{C}_{*}^{\mathrm{BM}, \bar{p}}(X ; R)$ for the complex of locally finite tame $\bar{p}$ intersection chains. Its homology $\mathfrak{H}_{*}^{\mathrm{BM}, \bar{p}}(X ; R)$ is the Borel Moore tame $\bar{p}$-intersection homology.

Let us recall the main properties of tame $\bar{p}$-intersection homology (see [5] or [12]). 
- It is natural with respect to stratum preserving maps, [5, Proposition 7.6].

- It satisfies Mayer-Vietoris property, [5, Proposition 7.10]. This comes from the fact that the classical subdivision operator $\mathrm{Sd}: C_{*}(X ; R) \rightarrow C_{*}(X ; R)$ can also be defined on the complex $\mathfrak{C}_{*}^{\bar{p}}(X ; R)$ and gives a quasi-isomorphism. As a consequence if we consider an open covering $\mathcal{U}$ of $X$ the inclusion of $\mathcal{U}$-small chains

$$
\mathfrak{C}_{*}^{\bar{p}, \mathcal{U}}(X ; R) \rightarrow \mathfrak{C}_{*}^{\bar{p}}(X ; R)
$$

is a quasi-isomorphism, [5, Corollaire 7.13].

- We also have a stratified homotopy invariance, [5, Proposition 7.7].

- Let $L$ be a compact filtered space, $\bar{p}$ a perversity on the open cone $\stackrel{\circ}{c} L$, of apex v. By denoting $\bar{p}$ the perversity induced on $L$, we have ([5, Proposition 7.9]):

$$
\mathfrak{H}_{k}^{\bar{p}}(\stackrel{\circ}{c} L ; R)= \begin{cases}\mathfrak{H}_{k}^{\bar{p}}(L ; R) & \text { if } k \leq D \bar{p}(\mathrm{v}), \\ 0 & \text { if } k>D p(\mathrm{v}) .\end{cases}
$$

- If $U$ is an open subset of a perverse space $(X, \bar{p})$ with the induced perversity, we define relative tame $\bar{p}$-intersection chains as the quotient $\mathfrak{C}_{*}^{\bar{p}}(X, U ; R)=$ $\mathfrak{C}_{*}^{\bar{p}}(X ; R) / \mathfrak{C}_{*}^{\bar{p}}(U ; R)$. Its homology denoted $\mathfrak{H}_{i}^{\bar{p}}(X, U ; R)$ fits into an exact sequence,

$$
\ldots \rightarrow \mathfrak{H}_{i}^{\bar{p}}(U ; R) \rightarrow \mathfrak{H}_{i}^{\bar{p}}(X ; R) \rightarrow \mathfrak{H}_{i}^{\bar{p}}(X, U ; R) \rightarrow \mathfrak{H}_{i-1}^{\bar{p}}(U ; R) \rightarrow \ldots
$$

Also if $K \subset U$ is compact, we have by excision $\mathfrak{H}_{i}^{\bar{p}}(X, X \backslash K ; R)=\mathfrak{H}_{i}^{\bar{p}}(U, U \backslash K ; R)$.

The next result connects tame $\bar{p}$-intersection chains to Borel-Moore ones.

Proposition 3.4. Let $(X, \bar{p})$ be a perverse space. Suppose that $X$ is locally compact, metrizable and separable. The complex of locally finite tame $\bar{p}$-intersection chains is isomorphic to the inverse limit of complexes,

$$
\mathfrak{C}_{*}^{\mathrm{BM}, \bar{p}}(X ; R) \stackrel{\cong}{\longrightarrow} \lim _{K \subset X} \mathfrak{C}_{*}^{\bar{p}}(X, X \backslash K ; R),
$$

where the limit is taken over all compact subsets of $X$.

Proof. Let $c$ be a chain of $\mathfrak{C}_{*}^{\mathrm{BM}, \bar{p}}(X ; R)$. Following [9, Proposition A.13] we know that there exists a locally finite family $\{\sigma\}$ of $\bar{p}$-intersection simplices, a locally family $\{\tau\}$ of $\bar{p}$-bad faces and a decomposition $c=\sum_{\sigma} r_{\sigma} \sigma+\sum_{\tau} c_{\tau}$, where $r_{\sigma} \in R$ and $c_{\tau}$ is the $\bar{p}$-intersection chain putting together all the simplices of $c$ having the same bad face $\tau$. In particular, any $c \in \mathfrak{C}_{*}^{\mathrm{BM}, \bar{p}}(X ; R)$ can be written as a locally finite decomposition

$$
c=\sum_{k \in J} c_{k},
$$

where each $c_{k}$ is a $\bar{p}$-intersection chain. Let $K$ be a compact subset of $X$. We define $J_{K}=\left\{k \in J \mid \operatorname{Supp}\left(c_{k}\right) \cap K \neq \emptyset\right\}$ and a map

$$
\rho_{K}: \mathfrak{C}_{*}^{\mathrm{BM}, \bar{p}}(X ; R) \rightarrow \mathfrak{C}_{*}^{\bar{p}}(X, X \backslash K ; R)
$$

by

$$
\rho_{K}(c)=\operatorname{class}_{K}\left(\sum_{k \in J_{K}} c_{k}\right) .
$$


This is a finite sum since $K$ is compact and $J$ is locally finite. Let us see that $\rho_{K}$ is well defined. If $c=\sum_{k \in J^{\prime}} e_{k}$ is another decomposition of $c$ as in (3.4) then the chain

$$
\sum_{k \in J_{K}} c_{k}-\sum_{k \in J_{K}^{\prime}} e_{k}=-\sum_{k \notin J_{K}} c_{k}+\sum_{k \notin J_{K}^{\prime}} e_{k}
$$

does not meet $K$ and therefore $\operatorname{class}_{K}\left(\sum_{k \in J_{K}} c_{k}\right)=\operatorname{class}_{K}\left(\sum_{k \in J_{K}^{\prime}} e_{k}\right)$.

Also, we observe that $\rho_{K}$ clearly commutes with differentials. By taking the limit over all compact subsets of $X$, we get a chain morphism,

$$
\rho: \mathfrak{C}_{*}^{\mathrm{BM}, \bar{p}}(X ; R) \rightarrow \lim _{K \subset X} \mathfrak{C}_{*}^{\bar{p}}(X, X \backslash K ; R) .
$$

If $\rho(c)=0$ then $\operatorname{class}_{K}(c)=0$ for any compact set $K$. This implies $c=0$ and therefore the injectivity of the map $\rho$.

In order to prove the surjectivity, we notice first that the topological hypotheses imply $([28,16 \mathrm{C}])$ the existence of an increasing sequence of compacts $K_{0} \subset K_{1} \subset \cdots K_{m} \subset \cdots$ such that any compact $K \subset X$ is included in some $K_{m}$. In particular, the family $\left(K_{m}\right)$ is cofinal in the family of compact subsets of $X$ and $X=\bigcup_{m \in \mathbb{N}} K_{m}$. Let us consider a collection of chains

$$
\mathfrak{c}=\left\{\mathfrak{c}_{m}=\operatorname{class}_{K}\left(c^{m}\right)\right\} \in \lim _{m} \mathfrak{C}_{*}^{\bar{p}}\left(X, X \backslash K_{m} ; R\right) .
$$

For $m \geq 0$, the natural inclusion $\mathfrak{c}_{m+1} \mapsto \mathfrak{c}_{m}$ gives $\rho_{K_{m}}\left(c^{m+1}\right)=\rho_{K_{m}}\left(c^{m}\right)$, that is, $\operatorname{Supp}\left(c^{m+1}-c^{m}\right) \cap K_{m}=\emptyset$. So, we have

$$
\operatorname{Supp}\left(c^{m+1}-c^{m}\right) \cap K_{m^{\prime}}=\emptyset \text { if } m^{\prime} \leq m .
$$

Let us consider the chain

$$
c=\sum_{k \in \mathbb{N}}\left(c^{k+1}-c^{k}\right) .
$$

Condition (3.5) gives $c \in \mathfrak{C}_{*}^{\mathrm{BM}, \bar{p}}(X ; R)$. We end the proof with $\rho(c)=\mathfrak{c}$, that is,

$$
\operatorname{class}_{K_{m}}\left(\sum_{k}\left(c^{k+1}-c^{k}\right)\right)=\operatorname{class}_{K_{m}}\left(c^{m}\right),
$$

for each $m \in \mathbb{N}$. Since $J_{K_{m}}=\left\{k \in \mathbb{N} \mid \operatorname{Supp}\left(c^{k+1}-c^{k}\right) \cap K_{m} \neq \emptyset\right\} \subset\{0, \ldots, m-1\}$ (cf. (3.5)) then

$$
\operatorname{class}_{K_{m}}\left(\sum_{k}\left(c^{k+1}-c^{k}\right)\right)=\operatorname{class}_{K_{m}}\left(\sum_{k<m}\left(c^{k+1}-c^{k}\right)\right)=\operatorname{class}_{K_{m}}\left(c^{m}\right) .
$$

Propositions 3.5 and 3.7 have been already established in the context of filtration depending perversities in [13].

Proposition 3.5. Let $L$ be a compact filtered space, $a \in \mathbb{N}$ and $\bar{p}$ a perversity on the product $\mathbb{R}^{m} \times L$. By still denoting $\bar{p}$ the perversity induced on $L$, we have

$$
\mathfrak{H}_{k}^{\mathrm{BM}, \bar{p}}\left(\mathbb{R}^{a} \times L ; R\right)=\mathfrak{H}_{k-a}^{\bar{p}}(L ; R) .
$$


Proof. We consider the following cofinal family of compact subsets of $\mathbb{R}^{a} \times L$,

$$
\left\{K_{n}=[-n, n]^{a} \times L \mid n \in \mathbb{N} .\right\} .
$$

As the open subsets $\left(\mathbb{R}^{a} \times L\right) \backslash K_{n}$ are stratified homeomorphic by the canonical inclusions, we are reduced to the case $n=0$. From Proposition 3.4, we deduce:

$$
\begin{aligned}
\mathfrak{H}_{k}^{\mathrm{BM}, \bar{p}}\left(\mathbb{R}^{a} \times L\right) & =\mathfrak{H}_{k}^{\mathrm{BM}, \bar{p}^{2}}\left(\mathbb{R}^{a} \times L,\left(\mathbb{R}^{a} \times L \backslash\{0\}\right) \times L\right)=\oplus_{i+j=k} H_{i}\left(\mathbb{R}^{a}, \mathbb{R}^{a} \backslash\{0\}\right) \otimes \mathfrak{H}_{j}^{\bar{p}}(L) \\
& =H_{a}\left(S^{a}\right) \otimes \mathfrak{H}_{k-a}^{\bar{p}}(L)=\mathfrak{H}_{k-a}^{\bar{p}}(L) .
\end{aligned}
$$

Before the next determination of Borel-Moore homology, we need the value of the intersection homology of a join with an $a$-dimensional sphere $S^{a}$. Let $X$ be a filtered space. Recall that the join $S^{a} * X$ is the quotient of the product with the closed unit ball, $B^{a+1} \times X$, by the equivalence relation $(z, x) \sim\left(z, x^{\prime}\right)$ if $z \in S^{a}$. The strata of $S^{a} * X$ are $S^{a}$ and the products $\stackrel{\circ}{B}^{a+1} \times S$, with $S$ a stratum of $X$ and $\stackrel{\circ}{B}^{a+1}$ the open unit ball. For any perversity on $X$, we get a perversity on the join from $\bar{p}\left(\stackrel{\circ}{B}^{a+1} \times S\right)=\bar{p}(S)$ and the choice of a number $\bar{p}\left(S^{a}\right)$.

Lemma 3.6. Let $\bar{p}$ be a perversity on the join $S^{a} * X$. We have

$$
\mathfrak{H}_{k}^{\bar{p}}\left(S^{a} * X ; R\right)=\left\{\begin{array}{cl}
\mathfrak{H}_{k}^{\bar{p}}(X ; R) & \text { if } k \leq D \bar{p}\left(S^{a}\right), \\
0 & \text { if } D \bar{p}\left(S^{a}\right)+1 \leq k \leq D \bar{p}\left(S^{a}\right)+a+1, \\
\mathfrak{H}_{k-a-1}^{\bar{p}}(X ; R) & \text { if } k \geq D \bar{p}\left(S^{a}\right)+a+2 .
\end{array}\right.
$$

Proof. Set $B_{1 / 2}^{a+1}=\left\{z \in \mathbb{R}^{a+1} \mid\|z\| \leq 1 / 2\right\}$ and $F=\left(B_{1 / 2}^{a+1} \times X\right) / \sim$. We apply the Mayer-Vietoris exact sequence to the open sets $U=\left(S^{a} * X\right) \backslash S^{a}$ and $V=\left(S^{a} * X\right) \backslash F$, $(3.6)$

$$
\ldots \longrightarrow \mathfrak{H}_{k}^{\bar{p}}(U \cap V) \stackrel{J_{k}}{\longrightarrow} \mathfrak{H}_{k}^{\bar{p}}(U) \oplus \mathfrak{H}_{k}^{\bar{p}}(V) \longrightarrow \mathfrak{H}_{k}^{\bar{p}}\left(S^{a} * X\right) \longrightarrow \mathfrak{H}_{k-1}^{\bar{p}}(U \cap V) \stackrel{J_{k-1}}{\longrightarrow} \ldots
$$

and get a short exact sequence

$$
0 \longrightarrow \text { Coker } J_{k} \longrightarrow \mathfrak{H}_{k}^{\bar{p}}\left(S^{a} * X\right) \longrightarrow \text { Ker } J_{k-1} \longrightarrow 0 \text {. }
$$

We study the map $J_{\ell}$ and for that determine first,

- $\mathfrak{H}_{\ell}^{\bar{p}}(U)=\mathfrak{H}_{\ell}^{\bar{p}}\left(\stackrel{\circ}{B}^{a+1} \times X\right)=\mathfrak{H}_{\ell}^{\bar{p}}(X)$,

- $\left.\left.\mathfrak{H}_{\ell}^{\bar{p}}(V)=\mathfrak{H}_{\ell}^{\bar{p}}\left(\left(S^{a} \times\right] 1 / 2,1\right] \times X\right) / \sim\right)=\mathfrak{H}_{\ell}^{\bar{p}}\left(S^{a} \times \stackrel{\circ}{\mathrm{c}} X\right)=\oplus_{i+j=\ell} H_{i}\left(S^{a}\right) \otimes \mathfrak{H}_{j}^{\bar{p}}(\stackrel{\circ}{ } X)$,

- $\mathfrak{H}_{\ell}^{\bar{p}}(U \cap V)=\mathfrak{H}_{\ell}^{\bar{p}}\left(S^{a} \times\right] 1 / 2,1[\times X)=\mathfrak{H}_{\ell}^{\bar{p}}\left(S^{a} \times X\right)=\oplus_{i+j=\ell} H_{i}\left(S^{a}\right) \otimes \mathfrak{H}_{j}^{\bar{p}}(X)$.

The map $J_{\ell}$ can be written as

$$
\oplus_{i+j=\ell} H_{i}\left(S^{a}\right) \otimes \mathfrak{H}_{j}^{\bar{p}}(X) \rightarrow \mathfrak{H}_{\ell}^{\bar{p}}(X) \oplus \oplus_{i+j=\ell} H_{i}\left(S^{a}\right) \otimes \mathfrak{H}_{j}^{\bar{p}}(\stackrel{\circ}{ }(X)
$$

which can be simplified in

$$
\mathfrak{H}_{\ell-a}^{\bar{p}}(X) \rightarrow \mathfrak{H}_{\ell}^{\bar{p}}(\stackrel{\circ}{\mathrm{c}} X) \oplus \mathfrak{H}_{\ell-a}^{\bar{p}}(\stackrel{\circ}{\mathrm{c} X}) .
$$

From (3.1), we deduce

$$
\begin{aligned}
& \operatorname{Ker} J_{\ell}=\left\{\begin{array}{cll}
0 & \text { if } \quad \ell \leq D \bar{p}\left(S^{a}\right)+a, \\
\mathfrak{H}_{\ell-a}^{\bar{p}}(X) & \text { if } \quad \ell>D \bar{p}\left(S^{a}\right)+a,
\end{array}\right. \\
& \text { Coker } J_{\ell}=\mathfrak{H}_{\ell}^{\bar{p}}(\stackrel{\circ}{ } X) \text {. }
\end{aligned}
$$


The result follows from (3.1) and (3.7).

Proposition 3.7. Let $L$ be a compact filtered space, $a \in \mathbb{N}$ and $\bar{p}$ a perversity on the open cone $\stackrel{\circ}{\mathrm{L}}$, of apex $\mathrm{v}$. By denoting $\bar{p}$ the perversity induced on $L$, we have

$$
\mathfrak{H}_{k}^{\mathrm{BM}, \bar{p}}\left(\mathbb{R}^{a} \times \stackrel{\circ}{\mathrm{c}} L ; R\right)= \begin{cases}0 & \text { if } k \leq a+D \bar{p}(\mathrm{v})+1, \\ \tilde{\mathfrak{H}}_{k-a-1}^{\bar{p}}(L ; R) & \text { if } k>a+D \bar{p}(\mathrm{v})+1 .\end{cases}
$$

Proof. We consider the following cofinal family of compact subsets of $\mathbb{R}^{a} \times{ }^{\circ} L$,

$$
\left\{K_{n}=[-n, n]^{a} \times \mathrm{c}_{n} L \mid n \in \mathbb{N}^{*}\right\} \cup\left\{K_{0}=\{(0, \mathrm{v})\}\right\},
$$

where $c_{n} L=\{[x, t] \in \stackrel{\circ}{c} L \mid t \leq n /(n+1)\}$. As the open subsets $\left(\mathbb{R}^{a} \times \stackrel{\circ}{\mathrm{c}} L\right) \backslash K_{n}$ are stratified homeomorphic by the canonical inclusions, we are reduced to the case $n=0$. From Proposition 3.4, we deduce:

$$
\mathfrak{H}_{k}^{\mathrm{BM}, \bar{p}}\left(\mathbb{R}^{a} \times \stackrel{\circ}{\mathrm{c}} L\right)=\mathfrak{H}_{k}^{\bar{p}}\left(\mathbb{R}^{a} \times \stackrel{\circ}{\mathrm{c}} L,\left(\mathbb{R}^{a} \times \stackrel{\circ}{\mathrm{c}} L\right) \backslash\{(0, \mathrm{v})\}\right) .
$$

Let $\mathrm{u}$ and $\mathrm{v}$ be the respective apex of $\stackrel{\circ}{\mathrm{c}}\left(S^{a-1} * L\right)$ and $\stackrel{\circ}{\mathrm{c}} L$. Let $\alpha:[0,1[\rightarrow[0, \infty[$ be a fixed homeomorphism. From [4, 5.7.4], we get the homeomorphism

$$
h: \stackrel{\circ}{\mathrm{c}}\left(S^{a-1} * L\right) \backslash\{\mathrm{u}\} \rightarrow\left(\mathbb{R}^{a} \times \stackrel{\circ}{\mathrm{c}} L\right) \backslash\{(0, \mathrm{v})\},
$$

given by

$$
h([z, y], r)=\left\{\begin{array}{ccc}
(2 r z,[y, \alpha(r)]) & \text { if } \quad\|z\| \leq 1 / 2 \\
(r z /\|z\|,[y, \alpha(2 r(1-\|z\|))]) & \text { if } \quad\|z\| \geq 1 / 2 .
\end{array}\right.
$$

Let us see that $h$ preserves the stratifications. We distinguish the following cases.

- $h\left(S^{a-1} \times\right] 0,1[)=\left(\mathbb{R}^{a} \times\{\mathrm{v}\}\right) \backslash\{(0, \mathrm{v})\}$ since $h([z, y], r)=(r z, \mathrm{v})$ if $\|z\|=1$.

- The restriction $\left.h: \stackrel{\circ}{B}^{a} \times L \times\right] 0,1\left[\rightarrow \mathbb{R}^{a} \times L \times\right] 0,1[$, given by

$$
(z, y, r) \mapsto\left\{\begin{array}{cl}
(2 r z, y, \alpha(r)) & \text { if } \quad\|z\| \leq 1 / 2 \\
(r z /\|z\|, y, \alpha(2 r(1-\|z\|))) & \text { if } \quad\|z\| \geq 1 / 2
\end{array}\right.
$$

is clearly a stratified homeomoprhism. The determination of the inclusion map

$$
\mathfrak{H}_{k}^{\bar{p}}\left(\left(\mathbb{R}^{a} \times \stackrel{\circ}{\mathrm{c}} L\right) \backslash\{(0, \mathrm{v})\}\right) \rightarrow \mathfrak{H}_{k}^{\bar{p}}\left(\mathbb{R}^{a} \times \stackrel{\circ}{\mathrm{c}} L\right)
$$

can thus be replaced by the determination of

$$
\mathfrak{H}_{k}^{\bar{p}}\left(\stackrel{\circ}{\mathrm{c}}\left(S^{a-1} * L\right) \backslash\{\mathrm{u}\}\right) \rightarrow \mathfrak{H}_{k}^{\bar{p}}(\stackrel{\circ}{\mathrm{c}} L) .
$$

Using Lemma 3.6 for the domain and (3.1) for the range, we obtain,

$$
\left\{\begin{array}{cll}
\mathfrak{H}_{k}^{\bar{p}}(L) \stackrel{\text { id }}{\rightarrow} \mathfrak{H}_{k}^{\bar{p}}(L) & \text { if } \quad k \leq D \bar{p}(\mathrm{v}), \\
0 \rightarrow 0 & \text { if } \quad D \bar{p}(\mathrm{v})+1 \leq k \leq D \bar{p}(\mathrm{v})+a, \\
\mathfrak{H}_{k-a}^{\bar{p}}(L) \rightarrow 0 & \text { if } \quad k \geq D \bar{p}(\mathrm{v})+a+1 .
\end{array}\right.
$$

The result follows from the long exact sequence of intersection homology.

Mention from [8, Proposition 6.7] the existence of cap products,

$$
\widetilde{N}_{\bar{q}}^{i}(X ; R) \otimes \mathfrak{C}_{j}^{\bar{p}}(X ; R) \stackrel{-\cap-}{\longrightarrow} \mathfrak{C}_{j-i}^{\bar{p}+\bar{q}}(X ; R)
$$

such that ([8, Proposition 6.6])

$$
(\eta \cup \omega) \cap \xi=\eta \cap(\omega \cap \xi) .
$$


Hence, the collection $\left\{\mathfrak{C}_{*}^{\bar{p}}(X ; R)\right\}_{\bar{p} \in \mathcal{P}}$ is a left perverse module over the perverse algebra $\left\{\widetilde{N}_{\bar{q}}^{*}(X ; R)\right\}_{\bar{q} \in \mathcal{P}}$. This cap product can also be extended in a cap product,

$$
\widetilde{N}_{\bar{q}}^{i}(X ; R) \otimes \mathfrak{C}_{j}^{\mathrm{BM}, \bar{p}}(X ; R) \stackrel{-\cap-}{\longrightarrow} \mathfrak{C}_{j-i}^{\mathrm{BM}, \bar{p}+\bar{q}}(X ; R),
$$

as follows. As (see (3.4)) any $c \in \mathfrak{C}_{*}^{\mathrm{BM}, \bar{p}}(X ; R)$ can be written as a locally finite sum of $\bar{p}$-intersection chains, $\xi=\sum_{k \in J} \xi_{k}$, we set

$$
\omega \cap \xi=\sum_{k \in J}\left(\omega \cap \xi_{k}\right) .
$$

A verification shows that this cap product is well defined and makes of $\left\{\mathfrak{C}_{*}^{\mathrm{BM}, \bar{p}}(X ; R)\right\}_{\bar{p} \in \mathcal{P}}$ a left perverse module over the perverse algebra $\left\{\widetilde{N}_{\bar{q}}^{*}(X ; R)\right\}_{\bar{q} \in \mathcal{P}}$.

3.2. Intersection cochains. The tame $\bar{p}$-intersection cohomology $\mathfrak{H}_{\bar{p}}^{*}(X ; R)$ is defined from the dual complex $\mathfrak{C}_{\bar{p}}^{*}(X ; R)=\operatorname{Hom}\left(\mathfrak{C}_{*}^{\bar{p}}(X ; R), R\right)$. We still denote $\mathfrak{d}$ its differential. This cohomology satisfies properties as those already listed for the tame homology (see [8]): naturality with respect to stratum preserving maps, Mayer-Vietoris property and invariance for stratified homotopy. Moreover, the case of an open cone on a compact perverse space is determined by

$$
\mathfrak{H}_{\bar{p}}^{k}(\stackrel{\circ}{c} L ; R)= \begin{cases}\mathfrak{H}_{\bar{p}}^{k}(L ; R) & \text { if } k \leq D p(\mathrm{v}), \\ \operatorname{Ext}\left(\mathfrak{H}_{k-1}^{\bar{p}}(L ; R), R\right) & \text { if } k=D \bar{p}(\mathrm{v})+1, \\ 0 & \text { if } k>D \bar{p}(\mathrm{v})+1\end{cases}
$$

The cap product (3.10) defines a map

$$
\star: \mathfrak{C}_{\bar{p}}^{j}(X ; R) \otimes \widetilde{N}_{\bar{q}}^{i}(X ; R) \longrightarrow \mathfrak{C}_{\bar{p}-\bar{q}}^{j+i}(X ; R)
$$

by

$$
(c \star \omega)(\xi)=c(\omega \cap \xi) .
$$

We check easily $c \star(\omega \cup \eta)=(c \star \omega) \star \eta$. Hence, the collection $\left\{\mathfrak{C}_{\bar{p}}^{*}(X ; R)\right\}_{\bar{p} \in \mathcal{P}}$ is a right perverse module over the perverse algebra $\left\{\widetilde{N}_{\bar{q}}^{*}(X ; R)\right\}_{\bar{q} \in \mathcal{P}}$. This operation respects the cochains with compact supports and makes $\left\{\mathfrak{C}_{\bar{p}, c}^{*}(X ; R)\right\}_{\bar{p} \in \mathcal{P}}$ a right perverse module over the perverse algebra $\left\{\widetilde{N}_{\bar{q}}^{*}(X ; R)\right\}_{\bar{q} \in \mathcal{P} \text {. }}$

There is also a notion of $\mathcal{U}$-small cochains relatively to an open covering, as follows.

Definition 3.8. Let $\mathcal{U}$ be an open covering of $X$ and $\bar{p}$ a perversity on $X$. The complex of $\mathcal{U}$-small cochains is $\mathfrak{C}_{\bar{p}}^{*}, \mathcal{U}(X ; R)=\operatorname{Hom}\left(\mathfrak{C}_{*}^{\bar{p}, \mathcal{U}}(X ; R), R\right)$. If $V$ is an open subset of $X$, we recall the category $\operatorname{Cov}(V)$ of Definition 2.5. The sheaf of intersection cochains $\mathbf{C}_{\bar{p}}^{*}$ is defined by its sections as the direct limit

$$
\Gamma\left(V ; \mathbf{C}_{\bar{p}}^{*}\right)=\lim _{\mathcal{U} \in \operatorname{Cov}(V)} \mathfrak{C}_{\bar{p}}^{*, \mathcal{U}}(V ; R) .
$$

Proposition 3.9. Let $(X, \bar{p})$ be a perverse space. The complex of sheaves $\mathbf{C}_{\bar{p}}^{*}$ over $X$ is a complex of soft and flat sheaves. 
Proof. (Softness) We have defined, on the complex of cochains $\mathfrak{C}_{\bar{p}}^{*}(X ; R)$, a structure of right module over $\widetilde{N}_{0}^{*}(X ; R)$. This structure being compatible with the restriction maps, the sheaf $\mathbf{C}_{\bar{p}}^{*}$ is a sheaf of right modules over $\widetilde{\mathbf{N}}_{\overline{0}}^{*}$. Thus the softness of $\widetilde{\mathbf{N}}_{\overline{0}}^{*}$ implies the softness of $\mathbf{C}_{\bar{p}}^{*}$.

(Flatness) The proof is similar to the proof made for $\widetilde{\mathbf{N}}_{\bar{p}}^{*}$ (see 2.6).

Mention also the existence in [16] of an intersection cohomology with compact supports defined by $\mathfrak{H}_{\bar{q}, c}^{*}(X ; R)=\lim _{K \text { compact }} \mathfrak{H}_{\bar{q}}^{*}(X, X \backslash K ; R)$. In [10], [8] we have connected these cohomologies with the blown-up cohomology. In particular, we have established the following isomorphisms.

Proposition 3.10. Let $(X, \bar{p})$ be a paracompact perverse pseudomanifold and $R$ a field. Then there are isomorphisms

$$
\mathscr{H}_{\bar{p}, c}^{*}(X ; R) \cong \mathfrak{H}_{D \bar{p}, c}^{*}(X ; R) \text { and } \mathscr{H}_{\bar{p}}^{*}(X ; R) \cong \mathfrak{H}_{D \bar{p}}^{*}(X ; R)
$$

3.3. Bidual. Let us take an injective resolution of $R$,

$$
0 \rightarrow R \rightarrow Q \stackrel{\rho}{\rightarrow} Q / R \rightarrow 0
$$

We denote by $I_{R}^{*}$ the cochain complex $I_{R}^{0}=Q \stackrel{\rho}{\longrightarrow} I_{R}^{1}=Q / R$, of differential $d=\rho$. We define a chain complex $\operatorname{Hom}_{*}\left(\mathfrak{C}_{\bar{p}, c}^{*}(X ; R), I_{R}^{*}\right)$, whose set $\operatorname{Hom}_{k}\left(\mathfrak{C}_{\bar{p}, c}^{*}(X ; R), I_{R}^{*}\right)$ of elements of degree $k$ is formed of the couples $\left(\varphi_{0}, \varphi_{1}\right)$ such that

$$
\varphi_{0}: \mathfrak{C}_{\bar{p}, c}^{k}(X ; R) \rightarrow Q \text { and } \varphi_{1}: \mathfrak{C}_{\bar{p}, c}^{k+1}(X ; R) \rightarrow Q / R,
$$

and whose differential is defined by $\mathbf{d}\left(\varphi_{0}, \varphi_{1}\right)=\left((-1)^{k} \varphi_{0} \circ \mathfrak{d},(-1)^{k} \varphi_{1} \circ \mathfrak{d}+d \circ \varphi_{0}\right)$.

The star product (3.12) defines a map

$$
\perp: \widetilde{N}_{\bar{q}}^{i}(X ; R) \otimes \operatorname{Hom}_{j}\left(\mathfrak{C}_{\bar{p}, c}^{*}, I_{R}^{*}\right) \rightarrow \operatorname{Hom}_{j-i}\left(\mathfrak{C}_{\bar{p}+\bar{q}, c}^{*}, I_{R}^{*}\right)
$$

by

$$
(\omega \perp \varphi)(c)=\varphi(c \star \omega) .
$$

We check easily $(\omega \cup \eta) \perp \varphi=\omega \perp(\eta \perp \varphi)$. Hence, the collection $\left\{\operatorname{Hom}_{*}\left(\mathfrak{C}_{\bar{p}, c}^{*}, I_{R}^{*}\right)\right\}_{\bar{p} \in \mathcal{P}}$ is a left perverse module over the perverse algebra $\left\{\widetilde{N}_{\bar{q}}^{*}(X ; R)\right\}_{\bar{q} \in \mathcal{P}}$.

The following result is the well-known "biduality" for intersection homology, proved in [1, Theorem V.8.10] in sheaf theory and in classical algebraic homology in [26], see also [22]. We give a short proof below.

Proposition 3.11. Let $(X, \bar{p})$ be a paracompact perverse pseudomanifold. Suppose that $X$ admits an exhaustive sequence of compacts $X=\cup_{i} K_{i}, K_{i} \subset \stackrel{\circ}{K}_{i+1}$, such that the $R$-module

$$
\mathfrak{H}_{\bar{p}}^{*}\left(X, X \backslash K_{i} ; R\right) \text { is finitely generated. }
$$

Then the morphism

$$
\Phi_{X}: \mathfrak{C}_{k}^{\mathrm{BM}, \bar{p}}(X ; R) \rightarrow \operatorname{Hom}_{k}\left(\mathfrak{C}_{\bar{p}, c}^{*}(X ; R), I_{R}^{*}\right),
$$


defined by $\Phi_{X}(\xi)=\left(\varphi(\xi)_{0}, 0\right)$ with $\varphi(\xi)_{0}(c)=c(\xi) \in R \subset Q$, is well defined and a quasi-isomorphism, linear for the structure of perverse modules over the perverse algebra $\left\{\widetilde{N}_{\bar{q}}^{*}(X ; R)\right\}_{\bar{q} \in \mathcal{P}}$

Remark 3.12. The hypothesis (3.16) is satisfied for PL-pseudomanifolds. This comes from the existence of isomorphisms between

- the intersection homology and its PL-version, see [12, Theorem 6.3.31],

- the PL-intersection homology and the simplicial intersection homology associated to a triangulation of $X,[12$, Theorem 6.3.30].

Proof of Proposition 3.11. From $\mathfrak{C}_{\bar{p}, c}^{*}(X ; R) \cong \lim _{\longrightarrow} \mathfrak{C}_{i} \mathfrak{C}_{*}^{\bar{p}}\left(X, X \backslash K_{i} ; R\right)$ and Proposition 3.4, we may replace $(3.17)$ by the map

$$
\psi_{X}: \lim _{K_{i}} \mathfrak{C}_{k}^{\bar{p}}\left(X, X \backslash K_{i} ; R\right) \rightarrow \lim _{K_{i}} \operatorname{Hom}_{k}\left(\mathfrak{C}_{*}^{\bar{p}}\left(X, X \backslash K_{i} ; R\right), I_{R}^{*}\right),
$$

defined as the limit of the maps

$$
\psi_{X, i}: \mathfrak{C}_{k}^{\bar{p}}\left(X, X \backslash K_{i} ; R\right) \rightarrow \operatorname{Hom}_{k}\left(\mathfrak{C}_{*}^{\bar{p}}\left(X, X \backslash K_{i} ; R\right), I_{R}^{*}\right)
$$

with $\psi_{X, i}(\xi)(c)=c(\xi) \in R$. Observe that the natural maps

$$
\operatorname{Hom}_{k}\left(\mathfrak{C}_{*}^{\bar{p}}\left(X, X \backslash K_{i+1} ; R\right), I_{R}^{*}\right) \rightarrow \operatorname{Hom}_{k}\left(\mathfrak{C}_{*}^{\bar{p}}\left(X, X \backslash K_{i} ; R\right), I_{R}^{*}\right)
$$

and

$$
\mathfrak{C}_{k}^{\bar{p}}\left(X, X \backslash K_{i+1} ; R\right) \rightarrow \mathfrak{C}_{k}^{\bar{p}}\left(X, X \backslash K_{i} ; R\right)
$$

are surjective. The Mittag-Leffler condition being satisfied, we have Milnor exact sequences ([27, Page 605]). Therefore, the map $\psi_{X}$ is a quasi-isomorphism if the $\psi_{X, i}$ 's are so. With the finiteness hypothesis on $\mathfrak{H}_{\bar{p}}^{*}\left(X, X \backslash K_{i} ; R\right)$ this last property is a classical result in homological algebra, see [22, Proposition 1.3] for instance.

\section{Poincaré and Verdier dualities}

In the case of a PL-pseudomanifold, we construct a commutative diagram of quasi-isomorphisms,

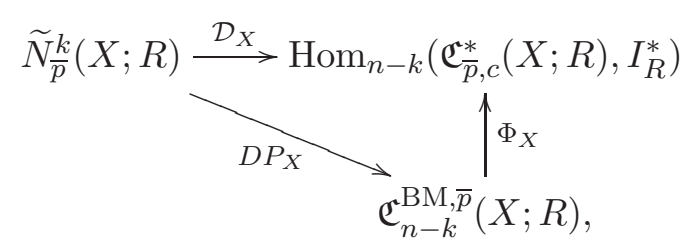

where $D P_{X}$ is the Poincaré morphism, defined by the cap product with a fundamental cycle and $\mathcal{D}_{X}$ is related to Verdier duality.

We first bring back definitions and basic properties of the elements of this diagram. Recall that $R$ is a principal ideal domain together with an injective resolution $I_{R}^{*}$. We refer to ([1, chapter V], [3], [20]) for more details on Verdier duality. For the construction of the quoted maps, we proceed along the next steps.

- In Proposition 4.2, we show that the Verdier dual sheaf $\mathbb{D}\left(\mathbf{C}_{\bar{p}}^{*}[n]\right)$ is quasi-isomorphic to the Deligne's sheaf. 
- If a quasi-isomorphism $\Psi_{\bar{p}}: \widetilde{\mathbf{N}}_{\bar{p}}^{*} \rightarrow \mathbb{D}\left(\mathbf{C}_{\bar{p}}^{*}[n]\right)$ is given, we show in Proposition 4.3 how one can pertube it in a $\widetilde{\mathbf{N}}_{*}^{*}$-linear map and keep a quasi-isomorphism.

- Finally, in Proposition 4.4, we construct the two quasi-isomorphisms $\mathcal{D}_{X}$ and $D P_{X}$ making the diagram (4.1) commutative.

This program is based on the existence of structures of perverse modules on the various complexes, see (3.15) and (3.11). Therefore any linear map (for these structures) of domain $\widetilde{N}_{\bar{p}}^{*}(X ; R)$ is entirely determined by the image of the constant map $1_{X}$.

Definition 4.1. Let $X$ be an oriented PL-pseudomanifold of dimension $n$ and $\mathbf{C}_{\bar{p}}^{*}$ the sheaf of $\bar{p}$-intersection cochains defined in (3.13). The Borel-Moore-Verdier dual of $\mathbf{C}_{\bar{p}}^{*}$ is the complex of sheaves $\mathbb{D}\left(\mathbf{C}_{\bar{p}}^{*}[n]\right)$ defined by

$$
U \mapsto \operatorname{Hom}_{n-k}\left(\Gamma_{c}\left(U ; \mathbf{C}_{\bar{p}}^{*}\right), I_{R}^{*}\right) .
$$

This is a cochain complex of sheaves.

Proposition 4.2. Let $X$ be an oriented PL-pseudomanifold of dimensionn. The complex of sheaves $\mathbb{D}\left(\mathbf{C}_{\bar{p}}^{*}[n]\right)$ is quasi-isomorphic to the Deligne's sheaf.

Proof. Let us prove that the complex of sheaves $\mathbb{D}\left(\mathbf{C}_{\bar{p}}^{*}[n]\right)$ is quasi-isomorphic to the Deligne sheaf $\mathbf{Q}_{\bar{p}}^{*}$. As the sheaf $\mathbf{C}_{\bar{p}}^{*}$ is flat and soft (Proposition 3.9), the sheaf $\mathbb{D}\left(\mathbf{C}_{\bar{p}}^{*}[n]\right)$ is an injective sheaf, hence it is soft. We are reduced to verify that the conditions of Definition 2.10 are fulfilled.

From Proposition 3.11, we may replace $\operatorname{Hom}_{n-k}\left(\mathfrak{C}_{\bar{p}, c}^{*}(X ; R), I_{R}^{*}\right)$ by $\mathfrak{C}_{n-k}^{\mathrm{BM}, \bar{p}}(X ; R)$. Now, if $U \cong \mathbb{R}^{n}$, we have $\oplus_{k} H_{n-k}^{\mathrm{BM}}(U)=H^{0}(U)$ and Property (1) is satisfied.

As in the proof of Theorem A, Property (2) is a consequence of $\mathfrak{H}_{n-i}^{\mathrm{BM}, \bar{p}}\left(\mathbb{R}^{n-k} \times \stackrel{\circ}{\mathrm{c}} L\right)=0$ if $i>\bar{p}(S)$. This last property has been established in Proposition 3.7.

The third condition is equivalent to the fact that the map $\mathfrak{H}_{n-i}^{\mathrm{BM}, \bar{p}}\left(\mathbb{R}^{n-k} \times \stackrel{\circ}{\mathrm{c}} L\right) \rightarrow$ $\mathfrak{H}_{n-i}^{\mathrm{BM}, \bar{p}}\left(\mathbb{R}^{n-k} \times(\stackrel{\circ}{\mathrm{c}} L \backslash\{\mathrm{v}\})\right.$ is a quasi-isomorphism for $i \leq \bar{p}(S)$. This is a consequence of Propositions 3.5 and 3.7.

The sheaves $\mathbb{D}\left(\mathbf{C}_{\bar{p}}^{*}[n]\right)$ and $\widetilde{\mathbf{N}}_{\bar{p}}^{*}$ are both quasi-isomorphic to Deligne's sheaves. As the sheaf $\mathbb{D}\left(\mathbf{C}_{\bar{p}}^{*}[n]\right)$ is injective there exists for any perversity $\bar{p}$ a (non unique) quasiisomorphism of sheaves $\boldsymbol{\chi}_{\bar{p}}: \widetilde{\mathbf{N}}_{\bar{p}}^{*} \rightarrow \mathbb{D}\left(\mathbf{C}_{\bar{p}}^{*}[n]\right)$. In the next proposition, we pertube it in a quasi-isomorphism compatible with the structures of perverse modules.

Proposition 4.3. Let $X$ be be an oriented PL-pseudomanifold of dimension $n$ and $U \subset$ $X$ an open subset. Let $\beta_{U}: \Gamma_{c}\left(U, \mathbb{D}\left(\mathbf{C}_{\overline{0}}^{*}[n]\right) \rightarrow I_{R}^{*}\right.$ be a cocycle in the class of $\chi_{\overline{0}}\left(1_{U}\right)$. For any perversity $\bar{p}$ on $X$, we construct a morphism of complex of sheaves

$$
\mathbf{\Psi}_{\bar{p}}: \widetilde{\mathbf{N}}_{\bar{p}}^{*} \rightarrow \mathbb{D}\left(\mathbf{C}_{\bar{p}}^{*}[n]\right)
$$

at the level of sections, $\Psi_{\bar{p}}: \Gamma\left(U ; \widetilde{\mathbf{N}}_{\bar{p}}^{*}\right) \rightarrow \Gamma_{c}\left(U, \mathbb{D}\left(\mathbf{C}_{\overline{0}}^{*}[n]\right)\right.$, by

$$
\Psi_{\bar{p}, U}(\omega)=\omega \perp \beta_{U} \text {. }
$$

Then $\mathbf{\Psi}_{\bar{p}}$ is a quasi-isomorphism, linear for the structure of perverse modules over the perverse algebra $\left\{\widetilde{N}_{\bar{q}}^{*}(X ; R)\right\}_{\bar{q} \in \mathcal{P}}$. 
Proof. By properties of Deligne sheaves it suffices to check if the restriction to the regular part $X_{\text {reg }}$ of $X$ is a quasi-isomorphism. Moreover, when restricted to the regular part, for any perversity $\bar{p}$ we have the following equality of complexes of sheaves

$$
\left(\widetilde{\mathbf{N}}_{\bar{p}}^{*}\right)_{\mid X_{\text {reg }}}=\left(\widetilde{\mathbf{N}}_{\overline{0}}^{*}\right)_{\mid X_{\text {reg }}} \text { and }\left(\mathbb{D}\left(\mathbf{C}_{\bar{p}}^{*}[n]\right)\right)_{\mid X_{\text {reg }}}=\left(\mathbb{D}\left(\mathbf{C}_{\overline{0}}^{*}[n]\right)\right)_{\mid X_{\text {reg }}} .
$$

Thus it is sufficient to consider the perversity $\overline{0}$.

Let $U \cong \mathbb{R}^{n}$ be an open subset of $X_{\text {reg. }}$. As $\Psi_{\overline{0}, U}\left(1_{U}\right)=1_{U} \perp \beta_{U}=\beta_{U}$ and $\beta_{U}$ is in the class of $\chi_{\overline{0}}\left(1_{U}\right)$, the difference $\Psi_{\overline{0}, U}\left(1_{U}\right)-\chi_{\overline{0}}\left(1_{U}\right)$ is a boundary. The map $\chi_{\overline{0}}$ being a quasi-isomorphism, the generator $\left[1_{U}\right]$ of $H^{0}\left(U ; \widetilde{\mathbf{N}}_{\overline{0}}^{*}\right)$ is sent on the generator $\left[\Psi_{\overline{0}, U}\left(1_{U}\right)\right]=\left[\chi_{\overline{0}}\left(1_{U}\right)\right]$ of $H^{0}\left(U ; \mathbb{D}\left(\mathbf{C}_{\overline{0}}^{*}[n]\right)\right)$. The result follows.

For the rest of this section, the space $X$ is an oriented, separable, metrizable $P L$ pseudomanifold of dimension $n$ and $\bar{p}$ is a perversity. Since the map $\Phi_{X}: \mathfrak{C}_{*}^{\mathrm{BM}, \overline{0}}(X ; R) \rightarrow$ $\operatorname{Hom}_{n-*}\left(\mathfrak{C}_{\overline{0}, c}^{*}(X ; R), I_{R}^{*}\right)$ is a quasi-isomorphism, there exists a cocycle $\gamma_{X} \in \mathfrak{C}_{n}^{\mathrm{BM}, \overline{0}}(X ; R)$ such that $\Phi_{X}\left(\gamma_{X}\right)-\chi_{\overline{0}}\left(1_{X}\right)$ is a boundary. The idea is to use Proposition 4.3 with $U=X$ and $\beta_{U}=\Phi_{X}\left(\gamma_{X}\right)$. More precisely, we define the two maps $\mathcal{D}_{X}$ and $D P_{X}$ by

$$
\mathcal{D}_{X}(\omega)=\omega \perp \Phi_{X}\left(\gamma_{X}\right) \text { and } D P_{X}(\omega)=\omega \cap \gamma_{X} .
$$

Proposition 4.4. The two maps $\mathcal{D}_{X}$ and $D P_{X}$ make commutative the diagram (4.1) and are quasi-isomorphisms.

Proof. We identify the homology of the sections $\Gamma\left(X ; \widetilde{\mathbf{N}}_{\bar{p}}^{*}\right)$ with the homology of $\widetilde{N}_{\bar{p}}^{*}(X ; R)$, and similarly for the homologies of $\Gamma\left(X ; \mathbb{D}\left(\mathbf{C}_{\bar{p}}^{*}[n]\right)\right)$ and $\operatorname{Hom}_{n-*}\left(\mathfrak{C}_{\bar{p}, c}^{*}(X ; R), I_{R}^{*}\right)$.

By construction we have $\mathcal{D}_{X}\left(1_{X}\right)=\Phi_{X}\left(D P_{X}\left(1_{X}\right)\right)$. As the three maps $D P_{X}, \mathcal{D}_{X}$ and $\Phi_{X}$ are compatible with the structure of perverse modules, we obtain the commutativiy of (4.1).

The maps $\Phi_{X}$ (see Proposition 3.11) and $\mathcal{D}_{X}$ (see Proposition 4.3) are quasi-isomorphisms. Thus $D P_{X}$ is one also.

The next statement is a direct consequence of Proposition 4.4 .

Corollary 4.5. The cap product with the fundamental class $\left[\gamma_{X}\right]$ induces an isomorphism between the blown-up intersection cohomology and the locally finite tame intersection homology,

$$
\mathscr{H}_{\bar{p}}^{k}(X ; R) \cong \mathfrak{H}_{n-k}^{\mathrm{BM}, \bar{p}}(X ; R) .
$$

From this corollary and [10, Theorem B], we deduce the existence of a commutative diagram between the two duality maps, corresponding to the blown-up cohomology and the blown-up cohomology with compact supports.

Corollary 4.6. Let $\gamma_{X} \in \mathfrak{C}_{n}^{\mathrm{BM}, \overline{0}}(X ; R)$ be a cycle representing the fundamental class. Then there is a commutative diagram whose vertical arrows are quasi-isomorphisms,

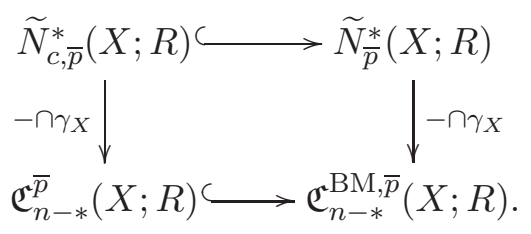




\section{REFERENCES}

[1] A. Borel and al., Intersection cohomology, Modern Birkhäuser Classics, Birkhäuser Boston Inc., Boston, MA, 2008, Notes on the seminar held at the University of Bern, Bern, 1983, Reprint of the 1984 edition. MR 2401086 (2009k:14046)

[2] A. Borel and J. C. Moore, Homology theory for locally compact spaces, Michigan Math. J. 7 (1960), 137-159. MR 0131271

[3] Glen E. Bredon, Sheaf theory, second ed., Graduate Texts in Mathematics, vol. 170, Springer-Verlag, New York, 1997. MR 1481706

[4] Ronald Brown, Topology and groupoids, BookSurge, LLC, Charleston, SC, 2006, Third edition of it Elements of modern topology [McGraw-Hill, New York, 1968; MR0227979], With 1 CD-ROM (Windows, Macintosh and UNIX). MR 2273730

[5] David Chataur, Martintxo Saralegi-Aranguren, and Daniel Tanré, Intersection Homology. General perversities and topological invariance, ArXiv 1602.03009. (To appear in Illinois J. of Math.) (2016).

[6] Steenrod squares on intersection cohomology and a conjecture of $M$ Goresky and W Pardon, Algebr. Geom. Topol. 16 (2016), no. 4, 1851-1904. MR 3546453

[7] Singular decompositions of a cap product, Proc. Amer. Math. Soc. 145 (2017), no. 8, 36453656. MR 3652815

[8] _ Blown-up intersection cohomology, An alpine bouquet of algebraic topology, Contemp. Math., vol. 708, Amer. Math. Soc., Providence, RI, 2018, pp. 45-102. MR 3807751

[9] , Intersection cohomology, simplicial blow-up and rational homotopy, Mem. Amer. Math. Soc. 254 (2018), no. 1214, viii+108. MR 3796432

[10] _ Poincaré duality with cap products in intersection homology, Adv. Math. 326 (2018), 314 $-351$.

[11] Pierre Deligne, Théorie de Hodge. II, Inst. Hautes Études Sci. Publ. Math. (1971), no. 40, 5-57. MR 0498551

[12] Greg Friedman, Singular intersection homology, Available at http://faculty.tcu.edu/gfriedman/index.html.

[13] Singular chain intersection homology for traditional and super-perversities, Trans. Amer. Math. Soc. 359 (2007), no. 5, 1977-2019 (electronic). MR 2276609 (2007k:55008)

[14] _ Intersection homology and Poincaré duality on homotopically stratified spaces, Geom. Topol. 13 (2009), no. 4, 2163-2204. MR 2507117 (2010d:55010)

[15] _ Intersection homology with general perversities, Geom. Dedicata 148 (2010), 103-135. MR 2721621

[16] Greg Friedman and James E. McClure, Cup and cap products in intersection (co)homology., Adv. Math. 240 (2013), 383-426.

[17] Mark Goresky and Robert MacPherson, Intersection homology theory, Topology 19 (1980), no. 2, 135-162. MR 572580 (82b:57010)

[18] Intersection homology. II, Invent. Math. 72 (1983), no. 1, 77-129. MR 696691 (84i:57012)

[19] Mark Hovey, Intersection homological algebra, New topological contexts for Galois theory and algebraic geometry (BIRS 2008), Geom. Topol. Monogr., vol. 16, Geom. Topol. Publ., Coventry, 2009, pp. 133-150. MR 2544388 (2010g:55009)

[20] Birger Iversen, Cohomology of sheaves, Universitext, Springer-Verlag, Berlin, 1986. MR 842190

[21] Henry C. King, Topological invariance of intersection homology without sheaves, Topology Appl. 20 (1985), no. 2, 149-160. MR 800845 (86m:55010)

[22] Erkki Laitinen, End homology and duality, Forum Math. 8 (1996), no. 1, 121-133. MR 1366538

[23] Guido Pollini, Intersection differential forms, Rend. Sem. Mat. Univ. Padova 113 (2005), 71-97. MR 2168981

[24] Frank Quinn, Homotopically stratified sets, J. Amer. Math. Soc. 1 (1988), no. 2, 441-499. MR 928266 (89g:57050)

[25] Martintxo Saralegi-Aranguren, de Rham intersection cohomology for general perversities, Illinois J. Math. 49 (2005), no. 3, 737-758. MR 2210257

[26] E. Spanier, Singular homology and cohomology with local coefficients and duality for manifolds, Pacific J. Math. 160 (1993), no. 1, 165-200. MR 1227511 
[27] George W. Whitehead, Elements of homotopy theory, Graduate Texts in Mathematics, vol. 61, Springer-Verlag, New York-Berlin, 1978. MR 516508

[28] Stephen Willard, General topology, Addison-Wesley Publishing Co., Reading, Mass.-London-Don Mills, Ont., 1970. MR 0264581

Lamfa, Université de Picardie Jules Verne, 33, Rue Saint-Leu, 80039 Amiens Cedex 1, FRANCE

E-mail address: David.Chataur@u-picardie.fr

Laboratoire de Mathématiques de Lens, EA 2462, Université D’Artois, SP18, Rue Jean Souvraz, 62307 Lens Cedex, France

E-mail address: martin.saraleguiaranguren@univ-artois.fr

Département de Mathématiques, UMR 8524, Université de Lille, 59655 Villeneuve D'Ascq Cedex, France

E-mail address: Daniel.Tanre@univ-lille.fr 